Pacific Journal of Mathematics

DDRR REGLLAR SEMMGROOU 


\title{
STANDARD REGULAR SEMIGROUPS
}

\author{
R. J. W ARNE
}

We give a structure theorem for a class of regular semigroups. Let $S$ be a regular semigroup, let $T$ denote the union of the maximal subgroups of $S$, and let $E(T)$ denote the set of idempotents of $T$. Assume $T$ is a semigroup (equivalently, $T$ is a semilattice $Y$ of completely simple semigroups $\left.\left(T_{y}: y \in Y\right)\right)$. If $Y$ has a greatest element and $e, f, g \in$ $E(T), e \geqslant f$, and $e \geqslant g$ imply $f g=g f$, we term $S$ a standard regular gemigroup. The structure of $S$ is given modulo right groups and an inverse semigroup $V$ in which every subgroup is a single element by means of an explict multiplication. We specialize the structure theorem to orthodox, $\mathscr{L}$-unipotent, and inverse semigroups, and to a class of semigroups with $Y$ an $\omega Y$-semilattice.

Finally, we show that $S$ is a regular extension of $T$ by $V$ in the sense of Yamada [19].

Let us first state the structure theorem. Let $Y$ be a semilattice with greatest element. Let $V$ be an inverse semigroup with semilattice of idempotents $Y$ such that each subgroup of $V$ consists of a single element. Let $(I, \circ)$ be a standard regular semilattice $Y$ of left zero semigroups $\left(I_{y}: y \in Y\right)$. Let $\left(J,^{*}\right)$ be a standard regular semilattice $Y$ of right groups $\left(J_{y}: y \in Y\right)$. Suppose $I_{y} \cap J_{y}=\left\{e_{y}\right\}$, a single idempotent element, and $e_{y}^{*} e_{z}=e_{y} \circ e_{z}=e_{y z}$ for all $y, z \in Y$. Let $H_{y}$ denote the maximal subgroup of $J_{y}$ containing $e_{y}$. Let $i \rightarrow B_{i}$ be a homomorphism of $(I, \circ)$ into $P(J)$, the semigroup of right translations of $(J, *)$; let $b \rightarrow \beta_{b}$ be a mapping of $V$ into End $\left(J,^{*}\right)$, the semigroup of endomorphism of $(J, *)$, and let $g$ be a mapping of $V \times V$ into $H=\mathrm{U}\left(H_{y}: y \in Y\right)$, a semilattice $Y$ of groups $\left(H_{y}: y \in Y\right)$ (with respect to the multiplication * in $J$ ) such that 1 (a) $j B_{i} \in H_{y z}$ for $i \in I_{y}$ and $j \in J_{z}$, (b) $J_{r} \beta_{b} \subseteq H_{b^{-1} r b}$, (c) $g(c, d) \in H_{(c d)-1}{ }_{c d}$. 2(a) $h B_{e_{y}}=h \beta_{y}=h^{*} e_{y}$ for $h \in J$ and $y \in Y$. (b) if $j \in H_{z}$ and $i \in I_{z}, j B_{i}=j$ (c) $g(y, z)=e_{y z}$ for $y, z \in Y$. 3(a)

$$
\beta_{c} \beta_{d}=\beta_{c d} C_{g(c, d)}\left(x C_{z}=z^{-1 *} x * z \text { for } x, z \in H\right)
$$

(b) $g(a, b c) * g(b, c)=g(a b, c) *\left(g(a, b) \beta_{c}\right)$. Let $(Y, I, J, V, B, \beta, g)$ denote $\left\{(i, a, j): a \in V, i \in I_{a a^{-1}}, j \in J_{a^{-1} a}\right\}$ under the multiplication (4)

$$
(i, a, j)(w, b, v)=\left(i \circ e_{(a b)(a b)^{-1}}, a b, g(a, b) * j B_{w} \beta_{b} * v\right) .
$$

We show (Theorem 3.14) that ( $Y, I, J, V, B, \beta, g)$ is a standard regular semigroup, and, conversely, every standard regular semigroup is isomorphic to some $(Y, I, J, V, B, \beta, g)$. 
If $X$ is a semigroup, $E(X)$ will denote the set of idempotents of $X$. Let $\mathscr{R}, \mathscr{L}, \mathscr{H}$, and $\mathscr{D}$ denote Green's relations (notation of [1]). Using [1, Theorem 2.3], $\mathscr{H}$ is the identity congruence on $V$. Hence, using a result of Munn [5, Theorem 2.3; see also 6], $V$ is isomorphic to a subsemigroup $U$ of the semigroup $X$ of isomorphisms between principal ideals of $E(V)$ with $E(U)=E(X)$.

In special cases, explicit multiplications for $V$ have been given (see for example [8] and [4]). Probably, the most familiar example of $V$ is the bicyclic semigroup.

The multiplication for $J$ is described by means of "connecting homomorphisms" between the $J_{y}$ (i.e. if $a \in J_{y}, b \in J_{z}, a * b=a \zeta_{y, y z} * b \zeta_{z, y z}$ where $\zeta_{y, w}(y \geqslant w)$ is a homomorphism of $J_{y}$ into $\left.J_{w}\right)$. The multiplication for $I$ is similarly characterized (see Remarks 1.7, 1.8, and 3.15).

A regular semigroup $X$ is termed locally inverse if $e, f, g \in E(X)$, $e \geqslant f$, and $e \geqslant g$ imply $f g=g f$. (Let $X$ be a regular semigroup and let $e \in E(X)$. Hence, if $a \in e X e$, there exists $y \in X$ such that $a=a y a=$ $(a e) y(e a)=a(e y e) a$. Thus, the semigroup $e X e$ is also regular. Hence, using [1, Theorem 1.17], a regular semigroup $X$ is locally inverse if and only if $e X e$ is an inverse semigroup for all $e \in E(X)$ ). Thus, a standard regular semigroup is a locally inverse semigroup such that $T$ is a semigroup and $Y$ has a greatest element.

Following Hall [3], a regular semigroup $X$ is termed orthodox if $E(X)$ is a semigroup. In general, a standard regular semigroup is not orthodox.

Yamada [18, Theorem 2] described the structure of locally inverse orthodox semigroups in terms of inverse semigroups (locally inverse orthodox $=$ generalized inverse in the sense of Yamada [18, Theorem 1]).

A Cliffordian semigroup is a semigroup which is a union of its subgroups. A semigroup $S$ is Cliffordian if and only if $S$ is a semilattice $Y$ of completely simple semigroups $\left(S_{y}: y \in Y\right)$ (Clifford, [1, Theorem 4.6]).

In $\S 1$, we show that the multiplication of a locally inverse Cliffordian semigroup $S$ is described by means of connecting homomorphisms between the $S_{y}$ (Theorem 1.6) and give some consequences of this theorem. The results of this section are applied repeatedly in the sequel.

In $\S \S 2$ and 3, we prove the converse and direct parts, respectively, of our structure theorem (Theorem 3.14).

Let $N$ denote the nonnegative integers and let $Y$ be a semilattice with greatest element. If $W=N \times Y$ with $(k, \alpha) \Lambda(s, \lambda)=(k, \alpha),(s, \lambda)$, or $(k, \alpha \Lambda \lambda)$ according to whether $k>s, s>k$, or $s=k$, we term $W$ an $\omega Y$-semilattice. A regular semigroup $S$ is termed $\omega Y$ - $\mathscr{L}$-unipotent if $E(S)$ is an $\omega Y$-semilattice of right zero semigroups $\left(E_{(n, \delta)}:(n, \delta) \in\right.$ 
$N \times Y)$ and $f_{(n, \delta)} \mathscr{D} f_{(m, \lambda)}\left(f_{(n, \delta)} \in E_{(n, \delta)} ; f_{(m, \lambda)} \in E_{(m, \lambda)}\right)$ if and only if $\delta=\lambda$. If $E_{(n, \delta)}$ is a single element for each $(n, \delta) \in N \times Y$, we term $S$ an $\omega Y$-inverse semigroup.

Munn [5, Theorem 3.3] described the structure of simple $\omega Y$ inverse semigroups. In [10, Theorem 4], Warne described the structure of simple $\omega Y$ - $\mathscr{L}$-unipotent semigroups such that $e \in E_{(n, \delta)}, f \in E_{\langle m, \lambda\rangle}$, and $(n, \delta)<(m, \lambda)$ implies $e<f$. When specialized to inverse semigroups, this result yields a theorem [10, Corollary 5] equivalent to Munn's theorem (see also [15, Lemma 2.1]). In [11, theorem and corollary], we show "simple" may be omitted. In [15, Theorem 6.1], we give a structure theorem for $\omega Y$ - $\mathscr{L}$-unipotent semigroups.

Let $S$ be a standard regular semigroup such that $T$ is an $\omega Y$ semilattice of completely simple semigroups $\left(T_{(n, \delta)}:(n, \delta) \in N \times Y\right)$. If $f_{(n, \delta)} \mathscr{D} f_{(m, \lambda)}\left(f_{(n, \delta)} \in E\left(T_{(n, \delta)}\right) ; f_{(m, \lambda)} \in E\left(T_{(m, \lambda)}\right)\right)$ if and only if $\delta=\lambda$, we term $S$ a standard regular semigroup of type $\omega Y$.

In $\S 4$, we specialize Theorem 3.14 to obtain the structure of standard regular semigroups of type $\omega Y$ (Theorem 4.2). In Theorem 4.2 , the factor terms " $g(c, d)$ " are omitted and $V$ is an $\omega Y$-inverse semigroup with each subgroup a single element. Hence, an explicit multiplication for $V$ is given by [15, Theorem 2.3]. Further specialization yields the structure of simple and bisimple standard regular semigroups of type $\omega$ ( $T$ is an $\omega$-chain of completely simple semigroups-no condition of the $\mathscr{D}$-classes).

In $\S 5$, we describe the structure of standard orthodox, standard $\mathscr{L}$-unipotent, and standard inverse semigroups (Theorems 5.1, 5.3, and 5.5 respectively). A standard regular semigroup is termed standard orthodox ( $\mathscr{L}$-unipotent)(inverse) if $T$ is a semilattice of rectangular groups (right groups)(groups). The structure theorems are obtained by specializing Theorem 3.14. In each of the theorems the term " $B$ ", is omitted. In Theorem 5.3 and 5.5, $I_{y}=\left\{e_{y}\right\}$ for each $y \in Y$. In Theorem 5.5, $J_{y}=H_{y}$ for each $y \in Y$.

Warne [9, page 206, paragraph 3] and Munn [5, page 66, paragraph 3] have exhibited inverse semigroups with identity on which $\mathscr{H}$, Green's relation, is not a congruence. Using Lemma 2.13, these semigroups are not standard.

Let $S$ be a standard regular semigroup. If $a \in S$, let $\mathscr{I}(a)$ denote the collection of inverses of $a$. Let $t=\left\{(a, b) \in S^{2}: a a^{\prime}, b b^{\prime} \in\right.$ $E\left(T_{y}\right)$ and $a^{\prime} a, b^{\prime} b \in E\left(T_{z}\right)$ for some $a^{\prime} \in \mathscr{J}(a), b^{\prime} \in \mathscr{J}(b)$, and $\left.y, z \in Y\right\}$.

In $\S 5$, we show $t$ is a congruence on $S, S / t \cong V$, $\operatorname{ker} t=T$, and $S$ is a regular extension of $T$ by $S / t$ in the sense of Yamada [19].

We use the definitions of Clifford and Preston [1] unless otherwise specified. In particular, $\mathscr{R}, \mathscr{L}, \mathscr{H}$, and $\mathscr{D}$ will denote Green's relations on a semigroup $S$, i.e., $(a, b) \in \mathscr{R}$ if $a \cup a S=b \cup b S ;(a, b) \epsilon$ $\mathscr{L}$ if $a \cup S a=b \cup S b ; \mathscr{H}=\mathscr{R} \cap \mathscr{L} ; \mathscr{D}=\mathscr{R} \circ \mathscr{L}((a, b) \in \mathscr{D}$ if there 
exists $x \in S$ such that $(a, x) \in \mathscr{R}$ and $(x, b) \in \mathscr{L})) . \quad R_{a}$ will denote the $\mathscr{R}$-class containing $a \in S$. A semigroup consisting of a single $\mathscr{D}$-class is termed a bisimple semigroup. A semigroup $S$ which is a union of a collection of pairwise disjoint subsemigroups $\left(S_{y}: y \in Y\right)$ where $Y$ is a semilattice and $S_{y} S_{z} \subseteq S_{y} \Lambda_{z}$ for all $y, z \in Y$ is termed a semilattice $Y$ of the semigroups $\left(S_{y}: y \in Y\right)$. If $Y=N$ with $n \Lambda m=\max (n, m), S$ is termed an $\omega$-chain of the semigroups $\left(S_{n}: n \in N\right)$. A semigroup $S$ is termed regular if $a \in a S a$ for all $a \in S$. If $S$ is a regular semigroup, for each $a \in S$, there exists $y \in S$ such that $a y a=a$ and $y a y=y$ (for example, if $a=a x a$, let $y=x a x$ [1, Lemma 1.14]). The element $y$ is termed an inverse of $a$. A regular semigroup $S$ is termed an inverse semigroup if each $a \in S$ has precisely one inverse. A rectangular band is the algebraic direct product of a left zero semigroup $U(x, y \in U$ implies $x y=x)$ and a right zero semigroup. A rectangular group is the algebraic direct product of a group and a rectangular band. A right group is a semigroup $X$ such that $a, b \in X$ implies there exists a unique $x \in X$ such that $a x=b$. If $S$ is a semigroup we may define a partial order " $\leqslant$ " on $E(S)$ by the rule: $e \leqq f$ means $e f=f e=e$. A band is a semigroup $S$ such that $x^{2}=x$ for each $x \in S$. If $S$ is a commutative band, $(S, \leqslant)$ is a semilattice with $a \Lambda b=a b$ and, conversely, every semilattice is a commutative band with $a b=a \Lambda b$ [1, Theorem 1.12]. A semigroup $S$ is termed simple if $S$ is its only ideal. If, furthermore, $e, f \in E(S)$ and $e \leqslant f$ imply $e=f, S$ is termed completely simple. The structure of such $S$ is known modulo groups by theorem of Rees [1, Theorem 3.5].

1. Locally inverse Cliffordian semigroups. In this section, we give a characterization of locally inverse Cliffordian semigroups (Theorem 1.6) and related results to be used in the sequel.

In the remainder of this section, $S$ will denote a locally inverse Cliffordian semigroup, i.e. $S$ is a locally inverse semilattice $Y$ of completely simple semigroups $\left(S_{y}: y \in Y\right)$.

LEMma 1.1. If $E \in E\left(S_{y}\right)$ and $y \geqslant z$, there exists precisely one $e \in E\left(S_{z}\right)$ such that $E \geqslant e$. Furthermore, $S_{z} L_{E} \subseteq L_{e}$ and $R_{E} S_{z} \subseteq R_{e}$.

Proof. If $y=z$, take $e=E$. Suppose $y>z$. Using the proof of [7, Theorem], there exists $e \in E\left(S_{z}\right)$ such that $E>e$. Let $g, h \in$ $E\left(S_{z}\right)$ with $g \leqq E$ and $h \leqq E$. Hence, since $S$ is locally inverse, $g h=h g$. Thus, $(h g)(h g)=h h g g=h g$, and, hence, $h g \in E\left(S_{z}\right)$. Furthermore, $g(h g)=h g=(h g) g$. Thus, $h g \leqslant g$. Thus, since $S_{z}$ is completely simple, $h g=g$. Similarly, $h g=h$ and, thus, $g=h$. The proof of the second sentence of the lemma is contained in the proof of [7, Theorem] for $y>z$. If $y=z$, apply the Rees theorem [1, 
Theorem 3.5].

Let $A \in S_{y}$ and suppose that $A \in R_{E} \cap L_{F}$ where $E, F \in E\left(S_{y}\right)$. Let $e$ and $f$ denote the unique idempotents of $S_{z}(y \geqslant z)$ under $E$ and $F$ respectively. We define $A \zeta_{y, z}=e A f$. It is shown in the proof of [7, Theorem] that $\zeta_{y, z}$ is well defined (i.e. $\zeta_{y, z}$ does not depend on the selection of $E$ and $F$ ).

LEMMA 1.2. For $y>z, \zeta_{y, z}$ is a homomorphism of $S_{y}$ into $S_{z}$. Let $A \in S_{y}$ and $B \in S_{z}$. If $y>z, A B=A \zeta_{y, z} B . \quad$ If $z>y, A B=A\left(B \zeta_{z, y}\right)$.

Proof. The proof of Lemma 1.2 is contained in the proof of [7, Theorem].

Lemma 1.3. If $y \in Y, \zeta_{y, y}$ is the identity mapping of $S_{y}$.

Proof. Let $A \in S_{y}$. Hence, $A \in R_{E} \cap L_{F}$ for some $E, F \in E\left(S_{y}\right)$. Let $e$ and $f$ denote the unique idempotents of $S_{y}$ under $E$ and $F$ respectively. Hence, since $S_{y}$ is completely simple, $e=E$ and $f=F$, Thus, $A \zeta_{y, y}=e A f=E A F=A$.

Let $y z=y \Lambda z$ in the semilattice $Y$.

Lemma 1.4. If $A \in S_{y}$ and $B \in S_{z}, A B=A \zeta_{y, y z} B \zeta_{z, y z}$.

Proof. Let $A \in L_{F}$ and $F \in E\left(S_{y}\right)$. Thus, utilizing Lemma 1.2 or 1.3, $A B=A(F B)=A \zeta_{y, y z}(F B)=A \zeta_{y, y z} F \zeta_{y, y z} B=(A F) \zeta_{y, y z} B=$ $A \zeta_{y, y z} B=A \zeta_{y, y z} B \zeta_{z, y z}$.

Lemma 1.5. For $x \geqslant y \geqslant z, \zeta_{x, y} \zeta_{y, z}=\zeta_{x, z}$.

Proof. Let $A \in S_{x}$ and suppose that $A \in R_{E} \in L_{F}$ for some $E, F \in$ $E\left(S_{x}\right)$. Let $e$ and $f$ denote the idempotents of $S_{y}$ under $E$ and $F$ respectively. Hence, $A \zeta_{x, y}=e A f$. By Lemma 1.1, $e A \in S_{y} L_{F} \subseteq L_{f}$ and $A f \in R_{E} S_{y} \subseteq R_{e}$. Let $e^{\prime}$ and $f^{\prime}$ denote the unique idempotents of $S_{z}$ under $e$ and $f$ respectively. Hence, $A \zeta_{x, y} \zeta_{y, z}=e^{\prime} e A f f^{\prime}=e^{\prime} A f^{\prime}$. However, $E \geqslant e \geqslant e^{\prime}$ and $F \geqslant f \geqslant f^{\prime}$. Hence, by Lemma 1.1, $A \zeta_{x, z}=$ $e^{\prime} A f^{\prime}=A \zeta_{x, y} \zeta_{y, z}$.

THEOREM 1.6. Let $\left\{S_{y}: y \in Y\right\}$ be a collection of pairwise disjoint completely simple semigroups indexed by the semilattice $Y$. For each $y, z \in Y$ with $y \geqslant z$, let $\zeta_{y, z}$ be a homomorphism of $S_{y}$ into $S_{y}$ such that

(1) $\zeta_{y, y}$ is the identity automorphism of $S_{y}$. 
(2) $\zeta_{x, y} \zeta_{y, z}=\zeta_{x, z}$ for $x \geqslant y \geqslant z$ in $Y$.

Let $S=\bigcup\left(S_{y}: y \in Y\right)$ and define a product on $S$ by the rule

(3) $A \circ B=A \zeta_{y, y z} B \zeta_{z, y z}$

where the right hand product is taken in $S_{y_{z}}$. Then, $(S, \circ)$ is a locally inverse Cliffordian semigroup.

Conversely, let $(S, \circ)$ be a locally inverse Cliffordian semigroup. Then, $S$ is the union of a collection of pairwise disjoint completely simple semigroups $\left(S_{y}: y \in Y\right)$ indexed by a semilattice $Y$. For each $y, z \in Y$ with $y \geqslant z$, there exists a homomorphism $\zeta_{y, z}$ of $S_{y}$ into $S_{z}$ such that (1) and (2) are valid and the multiplication is given by (3).

Proof. The converse is a consequence of [1, Theorem 4.6] and Lemmas 1.2-1.5. Let us now establish the direct part. Let $x y=$ $x A y$. Let $A \in S_{x}, B \in S_{y}$, and $C \in S_{z}$. Hence, uzing (3), the fact $\zeta_{y z, x y z}$ is a homomorphism, and (2).

$$
\begin{aligned}
A \circ(B \circ C) & =A \circ\left(B \zeta_{y, y z} C \zeta_{z, y z}\right)=A \zeta_{x, x y z}\left(\left(B \zeta_{y, y z} C \zeta_{z, y z}\right) \zeta_{y z, x y_{z}}\right) \\
& =A \zeta_{z, x y z}\left(B \zeta_{y, x y z} C \zeta_{z, x y z}\right) .
\end{aligned}
$$

Similarly, $(A \circ B) \circ C=\left(A \zeta_{x, x y z} B \zeta_{y, x y_{z}}\right) C \zeta_{z, x y z}$. Hence $(A \circ B) \circ C=A \circ(B \circ C)$ by associativity in $S_{x y_{z}}$. By (1) and (3), $S_{y}$ is a completely simple subsemigroup of $S$ for all $y \in Y$. Thus, $S_{y}$ is a Cliffordian semigroup for each $y \in Y$ by [1, Theorem 2.52]. Hence, $S$ is Cliffordian. Clearly, $S$ is a regular semigroup. Finally, let $E \in E\left(S_{x}\right), f \in E\left(S_{y}\right)$, and $g \in E\left(S_{z}\right)$ and suppose that $E \geqslant f$ and $E \geqslant g$. Hence, $x \geqslant y$ and $x \geqslant z$. Thus, using (3) and (1), $f=E \circ f=E \zeta_{x, y} f=f \circ E=f\left(E \zeta_{x, y}\right)$. Hence, $E \zeta_{x, y} \geqslant f\left(E \zeta_{x, y}, f \in E\left(S_{y}\right)\right)$. Thus, $f=E \zeta_{x, y}$ since $S_{y}$ is a completely simple semigroup. Similarly, $g=E \zeta_{x, z}$. Hence, $f \circ g=$ $f \zeta_{y, y z} g \zeta_{z, y z}=E \zeta_{x, y} \zeta_{y, y_{z}} E \zeta_{x, z} \zeta_{z, y_{z}}=E \zeta_{x, y z} E \zeta_{x, y z}=E \zeta_{x, y_{z}}$. Similarly, $g \circ f=$ $E \zeta_{x, y z}$. Thus, $g \circ f=f \circ g$, and hence, $S$ is locally inverse.

REMARK. In Theorem 1.6, we term $\left\{\zeta_{y, z}: y, z \in Y\right\}$ the collection of structure homomorphisms of $S$.

REMARK 1.7. In the statement of Theorem 1.6, we may replace "completely simple semigroup" by "left zero semigroup" and "Cliffordian semigroup" by "semilattice of left zero semigroups". Using Theorem 1.6, a band $E$ is left normal [17] if and only if $E$ is a locally inverse semilattice of left zero semigroups. Hence, we have obtained the Yamada-Kimura characterization of left normal bands [17, Theorem 1].

REMARK 1.8. In the statement of Theorem 1.6, we may replace "completely simple semigroup" by "right group" and "Cliffordian 
semigroup" by "semilattice of right groups".

The following result will be used in the sequel.

Proposition 1.9. Let $S$ be a locally inverse Cliffordian semigroup. Then, $\mathscr{L}, \mathscr{R}$, and $\mathscr{H}$ are congruence relations on $S$.

Proof. Let $S$ be a locally inverse Cliffordian semigroup. We first show that $\mathscr{L}$ is a congruence relation on $S$. We will apply Theorem 1.6 and its notation. Let $(x, y) \in \mathscr{L}$ in $S$. Hence, $(x, y) \in$ $\mathscr{L}$ in $S_{u}$ for some $u \in Y$. Let $z \in S_{v}$. Since $\zeta_{u, u v}$ is a homomorphism of $S_{u}$ into $S_{u v}, x \zeta_{u, u v} \mathscr{L} y \zeta_{u, u v}$ in $S_{u v}$. Using the Rees theorem [1, Theorem 3.5], $\mathscr{L}$ is a congruence relation on $S_{u v}$. Hence,

$$
z \circ x=z \zeta_{v, u v} x \zeta_{u, u v} \mathscr{L} z \zeta_{v, u v} y \zeta_{u, u v}=z \circ y \text {. }
$$

Thus, since $\mathscr{L}$ is a right congruence on any semigroup, $\mathscr{L}$ is a congruence on $S$. Similarly, $\mathscr{R}$ is a congruence relation on $S$.

2. Structure theorem for standard regular semigroups (proof of converse). In this section, we will use a sequence of twenty-one lemmas to establish the converse of our structure theorem for standard regular semigroups (Theorem 2.22).

Let $S$ be a standard regular semigroup and let $T$ denote the union of the maximal subgroups of $S$. Hence, $T$ is a semilattice $Y$ of completely simple semigroups $\left(T_{y}: y \in Y\right)$ where $Y$ has a greatest element $y_{0}$. Let $\left\{\zeta_{y, z}: y, z \in Y\right\}$ denote the set of structure homomorphisms of $T$. Let $E_{y}=E\left(T_{y}\right)$. Select and fix $e_{y_{0}} \in E_{y_{0}}$. For each $y \in Y$, define $e_{y}=e_{y_{0}} \zeta_{y_{0}, y}$. Let $S_{0}=e_{y_{0}} S e_{y_{0}}$.

LEMma 2.1. $E\left(S_{0}\right)=\left\{e_{y}: y \in Y\right\}$.

Proof. Since $e_{y_{0}} e_{y}=e_{y_{0}} \zeta_{y_{0}, y} e_{y}=e_{y}$, and, similarly, $e_{y} e_{y_{0}}=e_{y}, e_{y} \epsilon$ $E\left(S_{0}\right)$ for all $y \in Y$. Suppose $f \in E\left(S_{0}\right)$ and $f \in E_{y}$, say. Hence, $f \leqq e_{y_{0}}$ and $e_{y} \leqslant e_{y_{0}}$ implies $f e_{y}=e_{y} f$. Thus, since $T_{y}$ is completely simple, $f=e_{y}$.

LEMMA 2.2. $y \rightarrow e_{y}$ defines an isomorphism of $Y$ onto $E\left(S_{0}\right)$.

Proof. Let $y, z \in Y$. Hence,

$$
e_{y} e_{z}=e_{y} \zeta_{y, y z} e_{z} \zeta_{z, y z}=\left(e_{y_{0}} \zeta_{y_{0}, y} \zeta_{y, y_{z}}\right)\left(e_{y_{0}} \zeta_{y_{0}, z} \zeta_{z, y_{z}}\right)=e_{y z} .
$$

Lemma 2.3. $S_{0}=\bigcup\left(R_{e_{y}} \cap L_{e_{z}}: y, z \in Y\right)$.

Proof. Let $x \in R_{e_{y}} \cap L_{e_{z}}$ where $y, z \in Y$. Using Lemma 2.2, $e_{y_{0}} x=$ 
$e_{y_{0}} e_{y} x=e_{y} x=x$ and, similarly, $x e_{y_{0}}=x$. Conversely, if $x \in S_{0}$, let $x^{-1}$ denote the unique inverse of $x$ in $S_{0}$. Thus, using Lemma 2.1, $x x^{-1}=e_{y}$ and $x^{-1} x=e_{z}$ for some $y, z \in Y$. Hence, $x \in R_{e_{y}} \cap L_{e_{z}}$.

By the Rees theorem [1, Theorem 3.5], for $y \in Y, T_{y}=G_{y} \times$ $M_{y} \times N_{y}$ where $G_{y}$ is a group and $M_{y}$ and $N_{y}$ are sets under the multiplication $(g, i, j)(h, p, q)=\left(g f_{y}(j, p) h, i, q\right)$ where $(j, p) \rightarrow f_{y}(j, p)$ is a mapping of $N_{y} \times M_{y}$ into $G_{y}$. We note $e_{y}=\left(f_{y}\left(j_{y}, i_{y}\right)^{-1}, i_{y}, j_{y}\right)$, say, where $f_{y}\left(j_{y}, i_{y}\right)^{-1}$ is the inverse of $f_{y}\left(j_{y}, i_{y}\right)$ in the group $G_{y}$. Let $I_{y}$ denote the set of idempotents of the $\mathscr{L}$-class of $T_{y}$ containing $e_{y}$ and let $J_{y}$ denote the $\mathscr{R}$-class of $T_{y}$ containing $e_{y}$. Hence, $I_{y}=$ $\left\{\left(f_{y}\left(j_{y}, i\right)^{-1}, i, j_{y}\right) ; i \in M_{y}\right\}$ and $J_{y}=\left\{\left(g, i_{y}, j\right): g \in G\right.$ and $\left.j \in N_{y}\right\}$. Let $I=\mathrm{U}\left(I_{y}: y \in Y\right)$ and $J=\bigcup\left(J_{y}: y \in Y\right)$.

LEMMA 2.4. I is a standard regular semilattice $Y$ of left zero semigroups $\left(I_{y}: y \in Y\right)$.

Proof. Let $a \in I_{y}$ and $b \in I_{z}$. Thus, $a \mathscr{L} e_{y}$ and $b \mathscr{L} e_{z}$. Using Proposition 1.9 and Lemma 2.2, $a b \mathscr{L} e_{y_{z}}$. Furthermore, $a b a b=a b e_{y_{z}} a b=$ $a b e_{z} e_{y} a b=a b e_{z} e_{y} b=a b e_{y_{z}} b=a b b=a b$. Thus, $a b \in I_{y_{z}}$.

LEMMA 2.5. $J$ is a standard regular semilattice $Y$ of right groups $\left(J_{y}: y \in Y\right)$.

\section{Proof. Apply Proposition 1.9 and Lemma 2.2.}

The next two lemmas are special cases of left-right duals of [14, Lemmas 1.3 and 1.4]. Note our arbitrary representations of the " $e_{y}$ " requires a slight modification in the proof of [14, Lemma 1.3].

LEMMA 2.6. Every element of $T$ may be uniquely expressed in the form $x=i j$ where $i \in I_{y}$ and $j \in J_{y}$ for some $y \in Y$.

If $X$ is a set, $T_{X}$ will denote the semigroup (iteration) of mappings of $X$ into $X$.

LEMMA 2.7. There exists a mapping $j \rightarrow A_{j}$ of $J$ into $T_{I}$ and a mapping $p \rightarrow B_{p}$ of $I$ into $T_{J}$ such that $I_{y} A_{j} \leqq I_{y_{z}}$ for $j \in J_{z}$ and $J_{y} B_{p} \subseteq J_{y_{z}}$ for $p \in I_{z}$. If $j \in J$ and $p \in I, j p=p A_{j} j B_{p}$. Furthermore, $j p \mathscr{R} p A_{j}(\varepsilon T)$ and $j p \mathscr{L} j B_{p}(\varepsilon T)$.

LEMMA 2.8. $i A_{j}=e_{r s}$ for $i \in I_{r}$ and $j \in J_{s}$.

Proof. First, we show that $A_{j}=A_{e_{s}}$ for $j \in J_{s}$. Since $\mathscr{R}$ is a congruence relation on $T,\left(j, e_{s}\right) \in \mathscr{R}$ implies $\left(j i, e_{s} i\right) \in \mathscr{R}$ for all 
$i \in I$. Hence, using Lemma 2.7, $\left(i A_{j}, i A_{e_{s}}\right) \in \mathscr{R}$, for all $i \in I$. Thus, $i A_{j}=i A_{e_{s}}$ for $i \in I$. Let $i \in I_{r}$, say. Thus, since $e_{s} i \in I_{r s}$, we utilize Lemma 2.7 to obtain $\left(e_{s} i\right) e_{s r}=e_{s} i=i A_{e_{s}} e_{s} B_{i}$. Thus, by Lemmas 2.7 and 2.6, $i A_{e_{s}}=e_{s} i$. Since $i \mathscr{L} e_{r}, i \zeta_{r, r s} \mathscr{L} e_{r} \zeta_{r, r s}=e_{r s}$. Hence, $i \zeta_{r, r s} \in$ $I_{r s}$. Hence, using Theorem 1.6, $e_{s} i=e_{s r}\left(i \zeta_{r, s r}\right)=e_{s r}$. Thus, $i A_{j}=$ $i A_{e_{s}}=e_{s r}$.

Definition [1, p. 10]. A transformation $\rho$ of a semigroup $S$ is a right translation of $S$ if $(a b) \rho=a(b \rho)$ for all $a, b \in S$.

Lemma 2.9. For each $i \in I, B_{i}$ is a right translation of $J$.

Proof. Let $r \in J_{u}, s \in J_{v}$ and $x \in I_{p}$, say. Hence, utilizing Lemmas 2.5 and 2.7, $(r s) x=x A_{r s}(r s) B_{x}$ while $r(s x)=r\left(x A_{s} s B_{x}\right)=x A_{s} A_{r}\left(r B_{x A_{s}} s B_{x}\right)$. However, using Lemma 2.8, $r B_{x A_{s}}=r B_{e_{v p}}$. Using Lemma 2.5, $r e_{v p} \in$ $J_{u v p}$. Hence, $e_{u v p}\left(r e_{v p}\right)=r e_{v p}=e_{v p} A_{r} r B_{e_{v p}}$. Hence, using Lemmas 2.7 and 2.6, $r B_{e_{v p}}=r e_{v p}$. Thus, using Lemmas 2.7 and 2.6, $(r s) B_{x}=$ $r e_{v p} s B_{x}=r\left(s B_{x}\right)$.

LEMMA 2.10. $i \rightarrow B_{i}$ is a homomorphism of $I$ into $P(J)$, the semigroup of right translations of $J$.

Proof. Let $r, s \in I$ and $x \in J$. Thus, proceeding as in the proof of Lemma 2.9, $x(r s)=(r s) A_{x} x B_{r s}$ and

$$
(x r) s=r A_{x}\left(x B_{r} s\right)=\left(r A_{x} s A_{x B_{r}}\right)\left(x B_{r} B_{s}\right) .
$$

Thus $x B_{r s}=x B_{r} B_{s}$.

For each $y \in Y$, let $H_{y}$ denote the maximal subgroup of $S$ containing $e_{y}$.

Lemma 2.11. If $i \in I_{y}$ and $j \in J_{z}, j B_{i} \in H_{y z}$. If $j \in H_{y}, j B_{i}=j$.

Proof. Let $i \in I_{y}$ and $j \in J_{z}$. Since $j \mathscr{R} e_{z}, j \zeta_{z, y z} \mathscr{R} e_{z} \zeta_{z, y z}=e_{y z}$ and $j \zeta_{z, y z} \in J_{y z}$. Thus, $e_{y z} j i e_{y z}=e_{y z} j \zeta_{z, y z} i \zeta_{y, y z} e_{y z}=j \zeta_{z, y z} i \zeta_{y, y z}=j i$. Hence, since $j i \in T_{y z}, j i \in H_{y_{z}}$. However, using Lemmas 2.7 and 2.8, $j i=$ $i A_{j} j B_{i}=e_{y_{z}} j B_{i}=j B_{i}$. Thus, $j B_{i} \in H_{y_{z}}$. If $j \in H_{y}, j B_{i}=j i=j e_{y} i=$ $j e_{y}=j$.

Lemma 2.12. If $j \in J, j B_{e_{n}}=j e_{n}$.

Proof. Utilize the proof of Lemma 2.11.

LEMMA 2.13. Let $X$ be an inverse semigroup such that the union 
of the maximal subgroups of $X$ is a subsemigroup. Then, $\mathscr{H}$ is a congruence relation on $X$.

Proof. Let $\left.H=\mathrm{U}\left(H_{e}: e \in E(X)\right)\right)$. Using [1, Theorem 1.7], $H$ is an inverse semigroup which is a union of groups. Hence, $E(H)$ is contained in the center of $H$ by [1, Lemma 4.8]. Let $(a, b) \in \mathscr{H}$. Hence, $a a^{-1}=b b^{-1}$ and $a^{-1} a=b^{-1} b$. Let $c \in S$. Thus,

$$
\begin{aligned}
(b c)(b c)^{-1} & =b c c^{-1} b^{-1}=b a^{-1} a c c^{-1} a^{-1} a b^{-1}=b a^{-1}\left(a c c^{-1} a^{-1}\right) a b^{-1} \\
& =b a^{-1} a b^{-1} a c c^{-1} a^{-1}=a a^{-1} a c c^{-1} a^{-1}=(a c)(a c)^{-1}
\end{aligned}
$$

while $(b c)^{-1} b c=c^{-1} b^{-1} b c=c^{-1} a^{-1} a c=(a c)^{-1} a c . \quad$ Hence, $(a c, b c) \in \mathscr{H}$. Similarly, $(c a, c b) \in \mathscr{K}$.

LEMMA 2.14. $\mathscr{C}$ is a congruence relation on $S_{0}$.

Proof. Using Lemma 2.3, $\left\{H_{y}: y \in Y\right\}$ is the collection of maximal subgroups of $S_{0}$. Let $a \in H_{y}$ and $b \in H_{z}$, say. Since $a \mathscr{H} e_{y}, a \zeta_{y, y z} \mathscr{H} e_{y z}$ and, thus, $a \zeta_{y, y z} \in H_{y z}$. Similarly, $b \zeta_{z, y z} \in H_{y z}$. Thus, $a b=a \zeta_{y, y z} b \zeta_{z, y z} \in$ $H_{y z}$. Hence, using Lemma 2.13, $\mathscr{H}$ is a congruence relation on $S_{0}$.

LEMMA 2.15. There exists a homomorphism $\phi$ of $S_{0}$ onto an inverse semigroup $V$ where $E(V)=Y$ and each $\mathscr{H}$-class of $V$ consists of a single element. Furthermore, $(a, b) \in \mathscr{H}\left(\varepsilon S_{0}\right)$ if and only if $a \phi=b \phi$. Thus, if $h_{c}=c \phi^{-1},\left\{h_{c}: c \in V\right\}$ is the collection of $\mathscr{H}$ classes of $S_{0}$.

Proof. Using Theorem 2.14 and [1, Theorem 7.36], $S_{0 / \mathscr{C}}$ is an inverse semigroup. Let $a \rightarrow \bar{a}$ denote the natural homomorphism of $S_{0}$ onto $S_{0 / \%}$. Suppose $(\bar{a}, \bar{b}) \in \mathscr{H}\left(\varepsilon S_{0 / \mathscr{C}}\right)$. Hence, $\bar{a} \bar{a}^{-1}=\bar{b}^{-1}$ and $\bar{a}^{-1} \bar{a}=\bar{b}^{-1} \bar{b}$. Thus, $\overline{a a^{-1}}=\overline{b b^{-1}}$ and $\overline{a^{-1} a}=\overline{b^{-1} b}$. Hence, $\left(a a^{-1}, b b^{-1}\right) \epsilon$ $\mathscr{H}\left(\varepsilon S_{0}\right)$ and $\left(a^{-1} a, b^{-1} b\right) \in \mathscr{H}\left(\varepsilon S_{0}\right)$. Thus, $a a^{-1}=b b^{-1}$ and $a^{-1} a=b^{-1} b$. Hence, $(a, b) \in \mathscr{H}$, and, thus, $\bar{a}=\bar{b}$. Thus, each $\mathscr{H}$-class of $S_{0 / \mathscr{C}}$ consists of a single element. Using Lemma 2.2 and [1, Lemma 7.34], $\bar{e}_{y} \rightarrow y$ defines an isomorphism of $E\left(S_{0 / H}\right)$ onto the semilattice $Y$. Hence, we may extend this isomorphism to an isomorphism $\lambda$ of $S_{0 / \mathscr{C}}$ onto a semigroup $V$ with $E(V)=Y$. For $a \in S_{0}$, define $a \phi=\bar{a} \lambda$.

For each $c \in V$, select a representative element $v_{c} \in h_{c}$. For $y \in Y$, let $v_{y}=e_{y}$. Hence, using Lemma 2.15 and its proof, $v_{c} v_{c}^{-1}=e_{c c^{-1}}$ and $v_{c}^{-1} v_{c}=e_{c^{-1} c}$ for $c \in V$.

LEMMA 2.16. Every element of $S$ may be uniquely expressed in the form $i v_{c} j$ where $i \in I_{c c^{-1}}$ and $j \in J_{c^{-1} c_{c}}$. 
Proof. Let $x \in S$. Hence, $x \in R_{\varepsilon} \cap L_{f}$ where $e \in E_{y}$ and $f \in E_{z}$ for some $y, z \in Y$. Thus, $(e, i) \in \mathscr{R}$ for some $i \in I_{y}$ and hence $(x, i) \in$ $\mathscr{R}$. We note that $e_{y} \mathscr{D} e \mathscr{D} x \mathscr{D} f \mathscr{D} e_{z}$. Hence, $R_{e_{y}} \cap L_{e_{z}} \neq \square$. Thus, using Lemmas 2.3 and 2.15, $R_{e_{y}} \cap L_{e_{z}}=h_{c}$ for some $c \in V$. Thus, $v_{c} v_{c}^{-1}=e_{y}$ and $v_{c}^{-1} v_{c}=e_{z}$. Hence, $x=i x=i e_{y} x=i v_{c}\left(v_{c}^{-1} x\right)$. We will show that $v_{c}^{-1} x \in T_{c^{-1} c}$. Using the proof of [1, Theorem 2.18], there exists an inverse $x^{\prime}$ of $x$ such that $x^{\prime} \in R_{f} \cap L_{e}, x x^{\prime}=e$, and $x^{\prime} x=f$. Let $x x^{\prime}=\left(f_{y}(s, r)^{-1}, r, s\right)$ for some $r \in M_{y}$ and $s \in N_{y}$ and let $z=$ $\left(f_{y}\left(j_{y}, r\right)^{-1}, r, j_{y}\right)$. Using the Rees theorem, $e_{y} x x^{\prime} z=e_{y}$. Hence, $e_{z}=v_{c}^{-1} v_{c}=v_{c}^{-1} e_{y} v_{c}=v_{c}^{-1} e_{y} x x^{\prime} z v_{c}=v_{c}^{-1} x x^{\prime} z v_{c} \in v_{c}^{-1} x S$. However, $v_{c}^{-1} x=$ $e_{z} v_{c}^{-1} x \in e_{z} S$. Again, using the Rees theorem, $z e_{y} x x^{\prime}=x x^{\prime}$. Hence, $x=z e_{y} x=z v_{c} v_{c}^{-1} x$. Thus, $f=x^{\prime} x=x^{\prime} z v_{c} v_{c}^{-1} x \in S v_{c}^{-1} x$. However, $v_{c}^{-1} x \in S x=$ $S f$. Hence, $v_{c}^{-1} x \in R_{e_{z}} \cap L_{f} \leqq T_{z}$. Since $e_{z} v_{c}^{-1} x=v_{c}^{-1} x, v_{c}^{-1} x \in J_{z}$. Since $e_{z}=v_{c}^{-1} v_{c}, z=e_{z} \phi=\left(v_{c} \phi\right)^{-1} v_{c} \phi=c^{-1} c$. Thus, $v_{c}^{-1} x \in J_{c^{-1} c}$. Hence, $x=i v_{c} j$ where $i \in I_{c c^{-1}}$ and $j \in J_{c^{-1}}$. Suppose $i v_{c} j=r v_{d} s$ where $r \in I_{d d^{-1}}$ and $s \in J_{d^{-1} d}$. Since $j \mathscr{R} e_{c^{-1} c}, v_{c} j \mathscr{R} v_{c} e_{c^{-1} c}=v_{c} \mathscr{R} e_{c c^{-1}}$. Hence, $i v_{c} j \in$ $\mathscr{R} i e_{c c^{-1}}=i$. Similarly, $i v_{c} j \mathscr{L} j$. Hence, $i v_{c} j \in R_{i} \cap L_{j}$. Thus, $i \mathscr{R} r$ and $j \mathscr{L} s$. Hence, $c c^{-1}=d d^{-1}, i=r$, and $c^{-1} c=d^{-1} d$. Thus, $c \mathscr{H} d(\varepsilon V)$ and, hence, $c=d$. Thus, $i v_{c} j=i v_{c} s$. Therefore, $e_{c c^{-1}} i v_{c} j=e_{c c^{-1}} i v_{c} s$. Hence, $v_{c} j=v_{c} s$. Thus, $j=e_{c}^{-1}{ }_{c} j=v_{c}^{-1} v_{c} j=v_{c}^{-1} v_{c} s=e_{c}-1_{c} s=s$.

LEMMA 2.17. If $c, d \in V, v_{c} v_{d}=v_{c d} g(c, d)$ where $g$ is a function of $V \times V$ into $H=\bigcup\left(H_{y}: y \in Y\right)$ such that $g(c, d) \in H_{(c d)}{ }^{-1} c d . \quad$ If $y, z \in$ $Y, g(y, z)=e_{y z}$.

Proof. Using Lemma 2.16, $v_{c} v_{d}=i v_{x} j$ where $i \in I_{x x-1}$ and $j \in$ $J_{x^{-1} x}$. We first show $x=c d$. By the proof of Lemma 2.16, $i v_{x} j \in$ $R_{i} \cap L_{j}$. However, $\left.\left(v_{c} v_{d}\left(v_{c} v_{d}\right)^{-1}\right) \phi=(c d)(c d)^{-1}=e_{(c d)(c d)}\right)^{-1} \phi$. Hence, using Lemma 2.15, $\left(v_{c} v_{d}\right)\left(v_{c} v_{c}\right)^{-1}=e_{(c d)(c d)^{-1}}$. Similarly, $\left(v_{c} v_{d}\right)^{-1}\left(v_{c} v_{d}\right)=e_{(c d)^{-1}(c d)}$. Thus, $x x^{-1}=(c d)(c d)^{-1}$ and $x^{-1} x=(c d)^{-1} c d$. Thus, $(x, c d) \in \mathscr{H}(\varepsilon V)$. Hence, using Lemma 2.15, $x=c d$. Thus, $v_{c} v_{d}=i v_{c d} j$. Let $j=g(c, d)$. Hence, using Lemma 2.16, $g$ is a function of $V \times V$ into $J$ and $g(c, d) \in J_{(c d)^{-1}(c d)}$. Furthermore, $v_{c} v_{d}=e_{(c d)(c d)^{-1}} v_{c} v_{d}=e_{(c d)(c d)^{-1}} i v_{c d} g(c, d)=$ $v_{c d} g(c, d)$. We note that $g(c, d)=e_{(c d)-1(c d)} g(c, d)=v_{c d}^{-1} v_{c d} g(c, d)=$ $v_{c d}^{-1} v_{c} v_{d} \in S_{0}$. Thus, using Lemma 2.3, $g(c, d) \in H_{(c d)-1 c d}$. To obtain the last statement of the theorem, utilize Lemma 2.2

If $u \in J$ and $s \in V$, define $u \beta_{s}=v_{s}^{-1} u v_{s}$.

Lemma 2.18. For $s \in V, \beta_{s} \in$ End $J$ and $J_{r} \beta_{s} \subseteq H_{s^{-1} r s}$. Furthermore, if $j \in J$ and $s \in Y, j \beta_{s}=j e_{s}$

Proof. Let $j_{1}, j_{2} \in J$. Suppose $j_{1} \in J_{k}$ and $j_{2} \in J_{r}$. Hence, using Lemma 2.5, $j_{1} \beta_{s} j_{2} \beta_{s}=\left(v_{s}^{-1} j_{1} v_{s}\right)\left(v_{s}^{-1} j_{2} v_{s}\right)=v_{s}^{-1} j_{1} e_{s s^{-1}}\left(j_{2} e_{s s^{-1}}\right) v_{s}=v_{s}^{-1} j_{1} j_{2} v_{s}=$ $\left(j_{1} j_{2}\right) \beta_{s}$. Hence, $\beta_{s}$ is a homomorphism of $J$ into $S$. Since is a $J$ 
union of groups, $\beta_{s}$ is a homomorphism of $J$ into $T$. Since $e_{r} \beta_{s}=$ $v_{s}^{-1} e_{r} v_{s}=e_{s-1}, j \mathscr{R} e_{r}$ implies $j \beta_{s} \mathscr{R} e_{s-1 r s}$. Hence, $J_{r} \beta_{s} \leqq J_{s-1 r s}$. Thus, $\beta_{s} \in$ End $J$. Let $j \in J_{r}$. Thus, $v_{s}^{-1} j v_{s}=v_{s}^{-1} j v_{s} \cdot e_{s-1 s}=v_{s}^{-1} j v_{s} \cdot e_{s-1 s} \zeta_{s-1}{ }^{-1, s^{-1} r s}=$ $v_{s}^{-1} j v_{s} \cdot e_{s^{-1} r s}$. Thus $J_{r} \beta_{s} \subseteq H_{s^{-1} r s}$. If $s \in Y$ and $j \in J_{r}, j \beta_{s}=e_{s} j e_{s}=j e_{s}$.

If $j \in H$ and $z \in H$, define $j C_{z}=z^{-1} j z$. (Using Proposition 1.9 and Lemma 2.2, $H$ is a semilattice $Y$ of the groups $\left(H_{y}: y \in Y\right)$.)

Lemma 2.19. $\beta_{c} \beta_{d}=\beta_{c d} C_{g(c, d)}$.

Proof. Utilizing Lemma 2.17, $j \beta_{c} \beta_{d}=v_{d}^{-1} v_{c}^{-1} j v_{c} v_{d}=\left(v_{c} v_{d}\right)^{-1} j v_{c} v_{d}=$ $\left(v_{c d} g(c, d)\right)^{-1} j v_{c d} g(c, d)=j \beta_{c d} C_{g(c, d)}$.

LEMMA 2.20. $g(a, b c) g(b, c)=g(a b, c)\left(g(a, b) \beta_{c}\right)$.

Proof. Using Lemmas 2.17 and 2.5,

$$
\begin{aligned}
\left(v_{a} v_{b}\right) v_{c} & =v_{a b} g(a, b) v_{c}=v_{a b} g(a, b) e_{c c^{-1}} v_{c} \\
& =v_{a b} e_{c c^{-1}}\left(g(a, b) e_{c c^{-1}}\right) v_{c}=v_{a b} v_{c}\left(v_{c}^{-1} g(a, b) v_{c}\right) \\
& =v_{a b c} g(a b, c)\left(g(a, b) \beta_{c}\right)=e_{(a b c)(a b c)^{-1} v_{a b c} g(a b, c)\left(g(a, b) \beta_{c}\right) .}
\end{aligned}
$$

Using Lemmas 2.17 and 2.18, $g(a b, c)\left(g(a, b) \beta_{c}\right) \in J_{(a b c)-1_{a b c}}$. However,

$$
v_{a}\left(v_{b} v_{c}\right)=v_{a}\left(v_{b c} g(b, c)\right)=e_{(a b c)(a b c)-1} v_{a b c} g(a, b c) g(b, c) .
$$

We note that $g(a, b c) g(b, c) \in J_{(a b c)^{-1} a b c}$. Hence, using Lemma 2.16, $g(a, b c) g(b, c)=g(a b, c)\left(g(a, b) \beta_{c}\right)$.

If $a, b \in J$, define $a^{*} b=a b$. If $a, b \in I$, define $a \circ b=a b$.

LeMma 2.21. $S \cong\left\{(i, a, j): a \in V, i \in I_{a a^{-1}}\right.$, and $\left.j \in J_{a^{-1} a}\right\}$ under the multiplication $(i, a, j)(u, b, z)=\left(i \circ e_{(a b)(a b)-1}, a b, g(a, b)^{*} j B_{u} \beta_{3}^{*} z\right)$.

Proof. Let $i \in I_{a a^{-1}}, j \in J_{a^{-1} a}, u \in I_{b b^{-1}}$, and $z \in J_{b^{-1} b}$. Using Lemmas $2.7,2.8$, and 2.2 ,

$$
\begin{aligned}
\left(i v_{a} j\right)\left(u v_{b} z\right) & =i v_{a} u A_{j} j B_{u} v_{b} z \\
& =i v_{a} e_{b b^{-1} a^{-1} a} j B_{u} v_{b} z \\
& =i v_{a} e_{a^{-1} a} e_{b b^{-1}} j B_{u} v_{b} z \\
& =i v_{a} v_{b} v_{b}^{-1} j B_{u} v_{b} z \\
& =i v_{a b} g(a, b) j B_{u} \beta_{b} z \\
& =\left(i \circ e_{(a b)(a b)^{-1}}\right) v_{a b}\left(g(a, b)^{*} j B_{u} \beta_{b}^{*} z\right) .
\end{aligned}
$$

Using Lemmas 2.4, 2.5, 2.7, 2.17 and 2.18, $i \circ e_{(a b)(a b)^{-1}} \in I_{(a b)(a b)^{-1}}$ and $g(a, b)^{*} j B_{u} \beta_{b}^{*} z \in J_{(a b)^{-1} a b}$. Hence, using Lemma 2.16, $\left(i v_{a} j\right) \delta=(i, a, j)$ defines an isomorphism of $S$ onto the groupoid given in the statement 
of the lemma.

THEOREM 2.22. Let $S$ be a standard regular semigroup. Then, $S$ is isomorphic to $(Y, I, J, V, B, \beta, g)$ for some $Y, I, J, V, B, \beta, g$.

Proof. Utilize the remark before the proof of Lemma 2.21 and Lemmas 2.2, 2.4, 2.5, 2.10-2.12, 2.15, and 2.17-2.21.

REMARK 2.23. A semilattice $Y$ is said to be directed from above if $y, z \in Y$ implies there exists $w \in Y$ such that $w \geqslant y$ and $w \geqslant z$. Theorem 2.22 is valid if we replace the condition " $Y$ " has a greatest element by " $Y$ is directed from above" and "for each $y \in Y$, there exists $e_{y} \in E_{y}$ such that $e_{y} e_{z}=e_{y z}$ for all $y, z \in Y$ ". Just replace $S_{0}$ by the inverse semigroup $\bigcup\left(e_{y} S e_{y}: y \in Y\right)=\mathbf{U}\left(R_{e_{y}} \cap L_{e_{z}}: y, z \in Y\right)$ and note that for $z \leqslant y, e_{y} \zeta_{y, z}=e_{z}$.

3. Structure theorem for standard regular semigroups (proof of direct half). In this section, we show that $(Y, I, J, V, B, \beta, g)$ is a standard regular semigroup and establish other results to be used later in the sequel.

For brevity, let $S=(Y, I, J, V, B, \beta, g)$.

\section{LeMma 3.1. $S$ is a semigroup.}

Proof. Utilizing (4) and (1), closure is easily established. Let $(i, a, j)_{1}=i$ and $(i, a, j)_{3}=j$. Let $x=(i, a, j), y=(u, b, z)$, and $w=$ $(p, c, q)$. Using the fact $e_{r} \circ e_{s}=e_{r s}$ for all $r, s \in Y$ and (4), $((x y) w)_{1}=$ $(x(y w))_{1}$. Utilizing (4), the facts $\beta_{c} \in$ End $(J, *)$ and $i \rightarrow B_{i}$ is a homomorphism of $(I, \circ)$ into $P(J), 3(\mathrm{~b}), 3(\mathrm{a}), 1(\mathrm{c})$, the fact $e_{r}^{*} e_{s}=e_{r s}, 1(\mathrm{~b})$, and 2(a),

$$
\begin{aligned}
& ((x y) w)_{3}=g(a b, c)^{*}\left(g(a, b)^{*} j B_{u} \beta_{b}^{*} z\right) B_{p} \beta_{c}^{*} q \\
& =g(a b, c)^{*}\left(g(a, b) \beta_{c}\right) *\left(j B_{u} \beta_{b}^{*} z\right) B_{p} \beta_{c}^{*} q \\
& =g(a, b c)^{*} g(b, c)^{*} j B_{u} \beta_{b} \beta_{c}^{*} z B_{p} \beta_{c}^{*} q \\
& =g(a, b c)^{*} g(b, c)^{*}(g(b, c))^{-1} *\left(j B_{u} \beta_{b c}\right)^{*} g(b, c)^{*} z B_{p} \beta_{c}^{*} q \\
& =g(a, b c)^{*} e_{(b c)-1 b c}^{*} j B_{u} \beta_{b c}^{*} g(b, c)^{*} z B_{p} \beta_{c}^{*} q \\
& =g(a, b c)^{*} j B_{u} \beta_{b c}^{*} g(b, c)^{*} z B_{p} \beta_{c}^{*} q \\
& =g(a, b c)^{*} j B_{u} \beta_{b c}^{*} e_{(b c)(b c)^{-1}} \beta_{b c}^{*} g(b, c)^{*} z B_{p} \beta_{c}^{*} q \\
& =g(a, b c)^{*}\left(j B_{u}^{*} e_{(b c)(b c)-1}\right) \beta_{b c}^{*} g(b, c)^{*} z B_{p} \beta_{c}^{*} q \\
& =g(a, b c)^{*} j B_{u} B_{e(b c)(b c)-1} \beta_{b c}^{*} g(b, c)^{*} z B_{p} \beta_{c}^{*} q \\
& =g(a, b c)^{*} j B_{u^{\circ e(b c)(b c)-1}{ }^{-1}} \beta_{b c}^{*} g(b, c)^{*} z B_{p} \beta_{c}^{*} q \\
& =(x(y w))_{3} \text {. }
\end{aligned}
$$


Hence, $(x y) w=x(y w)$.

LEMMA 3.2. $E(S)=\left\{(i, a, j): a \in Y\right.$ and $\left.j B_{i}=e_{a}\right\}$.

Proof. Let $(i, a, j) \in E(S)$. Using 4, 2(a), 1(a), and 2(c), $a \in Y$ and $j B_{i}^{*} j=j$. Since $J_{a}$ is a right group, there exists $y \in J_{a}$ such that $j^{*} y=e_{a}$. Hence, using 1(a), $e_{a}=j^{*} y=j B_{i}^{*} j^{*} y=j B_{i}^{*} e_{a}=j B_{i}$. Conversely, if $a \in Y$ and $j B_{i}=e_{a}$, using (4), 2(c), and 2(a), $(i, a, j) \in$ $E(S)$.

Lemma 3.3. Let $(i, a, j),(u, b, v) \in S$. Then, $(i, a, j) \mathscr{R}(u, b, v)$ if and only if $i=u$.

Proof. First suppose that $i=u$. Hence, $a a^{-1}=b b^{-1}$. Let $x \in$ $I_{\left(a^{-1} b\right)\left(a^{-1} b\right)^{-1}}$. Thus, using (1) and the fact $J_{b^{-1 b}}$ is a right group, there exists $y \in J_{b^{-1} b}$ such that $\left(g\left(a, a^{-1} b\right)^{*} j B_{x} \beta_{a^{-1} b}\right)^{*} y=v$. Thus, using (4), $(i, a, j)\left(x, a^{-1} b, y\right)=(i, b, v)$. Similarly, there exists $p \in$ $I_{\left(b^{-1} a\right)\left(b^{-1} a\right)^{-1}}$ and $q \in J_{a^{-1} a}$ such that $(i, b, v)\left(p, b^{-1} a, q\right)=(i, a, j)$. Thus, $(i, a, j) \mathscr{R}(i, b, v)$. Conversely, suppose that $(i, a, j) \mathscr{R}(u, b, v)$. Using Thus, (4), $i \circ u=u$ and $u \circ i=i$. Hence, $i=u$.

LEMMA 3.4. $S$ is a regular semigroup.

Proof. Let $(i, a, j) \in S$. Using Lemma 3.3, $\left(i, a a^{-1}, e_{a a^{-1}}\right) \mathscr{R}(i, a, j)$. By $2(\mathrm{~b}), e_{a a^{-1}} B_{i}=e_{a a^{-1}}$. Hence, using Lemma 3.2, $\left(i, a a^{-1}, e_{a a^{-1}}\right) \in E(S)$. Thus each $\mathscr{R}$-class of $S$ contains an idempotent.

Lemma 3.5. Let $(i, a, j),(w, b, z) \in S$. Then, $(i, a, j) \mathscr{L}(w, b, z)$ if and only if $a^{-1} a=b^{-1} b$ and $(j, z) \in \mathscr{C}\left(\varepsilon J_{b^{-1}}\right)$.

Proof. We first show that $(i, a, j) \mathscr{L}(w, b, z)$ if $a^{-1} a=b^{-1} b$ and $(j, z) \in \mathscr{C}\left(\varepsilon J_{b^{-1} b}\right)$. Since $(j, z) \in \mathscr{H}\left(\varepsilon J_{b-1_{b}}\right)$, there exists $y \in H_{a^{-1} a}$ such that $y^{*} j=z$. Since $g\left(a^{-1}, a\right) \in H_{a^{-1} a}$ by $1(\mathrm{c})$, there exists $x \in H_{a^{-1} a}$ such that $x^{*} g\left(a^{-1}, a\right)^{*} j=z$. Using 1(b), 2(b), 3(a), 2(a), and 1(c), $g\left(a^{-1}, a\right)^{*} x \beta_{a-1} B_{i} \beta_{a}^{*} j=x^{*} g\left(a^{-1}, a\right)^{*} j=z$. Thus, $\left(e_{a^{-1} a}, a^{-1}, x \beta_{a^{-1}}\right)(i, a, j)=$ $\left(e_{a^{-1} a}, a^{-1} a, z\right)$. However, $\left(w, b,\left(g\left(b, b^{-1} b\right)\right)^{-1}\right)\left(e_{b^{-1}}, b^{-1} b, z\right)=(w, b, z)$. Hence, $\left(\left(w, b,\left(g\left(b, b^{-1} b\right)\right)^{-1}\right)\left(e_{a a}^{-1}, a^{-1}, x \beta_{a-1}\right)\right)(i, a, j)=(w, b, z)$. Similarly, there exists $p \in H_{b^{-1} b}$ such that $\left(\left(i, a,\left(g\left(a, a^{-1} a\right)\right)^{-1}\right)\left(e_{b^{-1}}, b^{-1}, p \beta_{b^{-1}}\right)\right)(w, b, z)=$ $(i, a, j)$. Hence, $(i, a, j) \mathscr{L}(w, b, z)$. Conversely, suppose that $(i, a, j) \mathscr{L}(w, b, z)$. Using (4) and (1), $a^{-1} a=b^{-1} b$ and $(j, z) \in \mathscr{H}\left(\varepsilon J_{b-1} b\right)$.

Lemma 3.6. Let $(i, a, j),(w, b, z) \in S$. Then, $(i, a, j) \mathscr{H}(w, b, z)$ if and only if $i=w, a=b$, and $(j, z) \in \mathscr{H}\left(\varepsilon J_{b}-1_{b}\right)$. 
Proof. Just note that each $\mathscr{\mathscr { C }}$-class of $V$ consists of a single element, and combine Lemmas 3.3 and 3.5.

REMARK. Lemmas 3.3 and 3.5 and their proofs were suggested by [16, Lemma 3.2] and its proof.

Lemma 3.7. Let $(i, a, j),(w, b, z) \in S$. Then, $(i, a, j) \mathscr{D}(w, b, z)$ if and only if $a \mathscr{D} b(\varepsilon V)$.

Proof. Suppose that $a \mathscr{D} b(\varepsilon V)$. Hence, there exists $x \in V$ such that $a \mathscr{R} x$ and $x \mathscr{L} b$. Hence, $a a^{-1}=x x^{-1}$ and $b^{-1} b=x^{-1} x$. Thus, using Lemmas 3.3 and $3.5,(i, a, j) \mathscr{R}(i, x, z) \mathscr{L}(w, b, z)$. Conversely, if $(i, a, j) \mathscr{D}(w, b, z)$, using (4), $a \mathscr{D} b(\varepsilon V)$.

LEMMA 3.8. $S$ is a bisimple semigroup if and only if $V$ is a bisimple semigroup.

\section{Proof. Apply Lemma 3.7.}

Lemma 3.9 For each $y \in Y$, let $T_{y}=\left\{(i, y, j): i \in I_{y}\right.$ and $\left.j \in J_{y}\right\}$. Then, $T_{y}$ is a completely simple semigroup.

Proof. First, we show that $T_{y}$ is a simple semigroup. Let $(i, y, j),(u, y, v) \in T_{y}$. Hence, using 1(a), 2(a), and 2(c), $(i, y, j)(u, y, v)$ $\left(i, y, j B_{u}^{*} v\right) \in T_{y}$. Since $J_{y}$ is a right group, there exists $x \in J_{y}$ such that $j^{*} x=v$. Hence, using $2(\mathrm{~b})$ and $2(\mathrm{a})$,

$$
\left(u, y, e_{y}\right)(i, y, j)\left(e_{y}, y, x\right)=(u, y, v) .
$$

Next, we show $T_{y}$ is completely simple. Let $e, f \in E\left(T_{y}\right)$ and suppose that $e \leqslant f$. Hence, using Lemma 3.2, $e=(i, y, j)$ and $f=(w, y, z)$, say, where $j B_{i}=z B_{w}=e_{y}$. Thus, $(w, y, z)(i, y, j)=(i, y, j)$ implies $w=i$. Hence, $(i, y, j)(i, y, z)=(i, y, z)=(i, y, j)$. Thus, $z=j$ and, hence, $(w, y, z)=(i, y, j)$.

Lemma 3.10. Let $T=\mathrm{U}\left(T_{y}: y \in Y\right)$. Then, $T$ is a semilattice $Y$ of the completely simple semigroup $\left(T_{y}: y \in Y\right)$.

Proof. Apply (4), 2(c), 1(a), and 2(a).

LEMMA 3.11. $T$ is the union of the maximal subgroups of $S$.

Proof. If $x \in T_{y}, x$ is contained in some subgroup of $S$ (each completely simple semigroup is a union of its subgroups by [1, Theorem 2.52]). Thus, $x \in H_{e}$ for some $e \in E(S)$. Hence, $T \subseteq X$, the 
union of the maximal subgroups of $S$. If $c \in X, c \mathscr{H} e$ for some $e \in$ $E(S)$. Hence, $e=(i, y, j)$ for some $y \in Y$ by Lemma 3.2. Thus, using Lemma 3.6, $c \in T_{y}$.

\section{LEMMA 3.12. $T$ is a locally inverse semigroup.}

Proof. Let $(i, a, j),(u, b, v)$, and $(w, c, z) \in E(S)$ such that $(i, a, j) \geqslant$ $(u, b, v)$ and $(i, a, j) \geqslant(w, c, z)$. Hence, $(i, a, j)(u, b, v)=(u, b, v)(i, a, j)=$ $(u, b, v)$. Using Lemma 3.2, $a, b \in Y$, and, hence, $a \geqslant b$. Since $i \circ e_{b}=$ $u, i \circ u=i \circ\left(i \circ e_{b}\right)=i \circ e_{b}=u$ while $u \circ i=u \circ(u \circ i)=u$. Hence, $i \geqslant u$. Using (4), 2(c), 2(a) and 1(a), $j B_{u}^{*} v=v B_{i}^{*} j=v$. Hence, using the fact $j B_{u} \in H_{b}$ and $v \in J_{b}$, a right group, $j B_{u}=e_{b}$. Hence, using Lemma 3.2, $e_{b}=v B_{u}=\left(v B_{i}^{*} j\right) B_{u}=v B_{i}^{*} j B_{u}=v B_{i}^{*} e_{b}=v B_{i}$. Thus, $e_{b}^{*} j=v$. Let $\left\{\zeta_{p, q}: p, q \in Y\right\}$ denote the set of structure homomorphisms of $\left(J,{ }^{*}\right)$. Thus, $e_{b}{ }^{*} j \zeta_{a, b}=v$. Hence, $j \zeta_{a, b}=v$. We have shown that $(i, a, j) \geqslant$ $(u, b, v)$ implies $i \geqslant u, a \geqslant b$, and $v=j \zeta_{a, b}$. Similarly, $(i, a, j) \geqq(w, c, z)$ implies $i \geqslant w, a \geqslant c$, and $z=j \zeta_{a, c}$. Since $(I, \circ)$ is a locally inverse semigroup, $u \circ w=w \circ u$. Using 2(c), 2(a), and 1(a), $(u, b, v)(w, c, z)=$ $\left(u \circ e_{b c}, b c, v B_{w}^{*} z\right)$ while $(w, c, z)(u, b, v)=\left(w \circ e_{b c}, b c, z B_{u}^{*} v\right)$. Let $\left\{\phi_{p, q}\right.$ : $p, q \in Y\}$ denote the set of structure homomorphisms of $(I, \circ)$. Hence, $w \circ u=w \phi_{c, b c} \circ u \dot{\phi}_{b, b c}=w \dot{\phi}_{c, b c} \circ e_{b c} \circ u \dot{\phi}_{b, b c}=w \circ e_{b c}$. Similarly, $u \circ w=u \circ e_{b c}$. Hence, $u \circ e_{b c}=w \circ e_{b c}$. Furthermore,

$$
\begin{aligned}
v B_{w}^{*} z & =v B_{w}^{*} e_{b c}^{*} z=v B_{w} B_{e_{b c}}^{*} z \\
& =v B_{w \circ e_{b c}} * z=v B_{u \circ e_{b c}} * z \\
& =v B_{u}^{*} e_{b c}^{*} z=e_{b}^{*} e_{b c}^{*} z \\
& =e_{b c}^{*} z=z \zeta_{c}, b c .
\end{aligned}
$$

Similarly, $z B_{u}^{*} v=v \zeta_{b, b c}$. Hence, $v B_{w}^{*} z=j \zeta_{a, c} \zeta_{c, b c}=j \zeta_{a, b c}=j \zeta_{a, b} \zeta_{b, b c}=$ $v \zeta_{b, b c}=z B_{u}^{*} v$. Thus, $(u, b, v)(w, c, z)=(w, c, z)(u, b, v)$.

Theorem 3.13. ( $Y, I, J, V, B, \beta, g)$ is a standard regular semigroup.

Proof. Utilize Lemmas 3.1, 3.4, and 3.10-3.12.

Theorem 3.14. ( $Y, I, J, V, B, \beta, g)$ is a standard regular semigroup and, conversely, every standard regular semigroup is isomorphic to some $(Y, I, J, V, B, \beta, g)$.

Proof. Combine Theorems 2.22 and 3.13 .

REMARK 3.15. Let $J$ and $H$ be as in the statement of Theorem 3.14. Using the proof of [1, Theorem 1.27], Theorem 1.6, and Pro- 
position $1.9, J \cong \bigcup\left(H_{y} \times E\left(J_{y}\right): y \in Y\right)$ where, if $a \in H_{y}, c \in H_{z}, b \in$ $E\left(J_{y}\right)$, and $d \in E\left(J_{z}\right),(a, b)(c, d)=(a c, b d) \in H_{y z} \times E\left(J_{y z}\right)$. The multiplications in $H$ and $E(J)$ are given by the corresponding specializations of Theorem 1.6. These specializations yeild theorems of Clifford [1, Theorem 4.11] and Yamada and Kimura [17, Theorem 1] respectively.

REMARK 3.16. Let $e_{y}=\left(e_{y}, y, e_{y}\right)$. Hence, using (4), 2(c), 2(a), $e_{y} e_{z}=e_{y z}$. Thus, if we replace " $Y$ has a greatest element" by " $Y$ is directed from above" in the definition of $(Y, I, J, V, B, \beta, g)$, we obtain the semigroup of Remark 2.23. Hence, Theorem 3.14 with appropriate modifications characterizes these semigroups.

4. Standar dregular semigroups of type $\omega Y$. Let $S$ be a regular semigroup such that $T$ is a locally inverse $\omega Y$-semilattice $A$ of completely simple semigroups $\left\{T_{(n, \delta)}:(n, \delta) \in A\right\}$. If $f_{(n, \delta)} \mathscr{D} f_{(m, \lambda)}\left(f_{(n, \delta)} \in\right.$ $\left.E_{(n, \delta)}: f_{(m, \lambda)} \in E_{(m, \lambda)}\right)$ if and only if $\delta=\lambda$, we term $S$ a standard regular semigroup of type $\omega Y$. If $\delta_{0}$ is the greatest element of $Y,\left(\circ, \delta_{0}\right)$ is the greatest element of $A$. We give a characterization of standard regular semigroups of type $\omega Y$ (Theorem 4.2). A regular semigroup $S$ such that $T$ is a locally inverse $\omega$-chain of completely simple semigroups $\left(T_{n}: n \in N\right)$ (no further condition) is termed a standard regular semigroup of type $\omega$. We show that $S$ is a simple (bisimple) standard regular semigroup of type $\omega$ if and only if $S$ is a standard regular semigroup of type $\omega Y$ with $Y$ a finite chain (a single element) (Theorem 4.3) (Theorem 4.4). Hence, the structure of these semigroups is given by specializing Theorem 4.2.

To establish Theorem 4.2, we use a more general result on "split" extensions (Theorem 4.1).

Let $S$ be a standard regular semigroup. In the notation of $\S 2$, let $\left\{h_{c}: c \in V\right\}$ denote the collection of $\mathscr{H}$-classes of $S_{0}$. For each $c \in V$, select $v_{c} \in h_{c}$. If $v_{c} v_{d}=v_{c d}$ for all $c, d \in V$, we term $S$ a split extension of $T$ by $V$.

Let $Y, V, I, J, H$, and $\left\{e_{k}\right\}$ be as in the definition of $(I, J, V, B, \beta, g)$. Let $i \rightarrow B_{i}$ be a homomorphism of $(I, \circ)$ into $P(J)$, and let $v \rightarrow \beta_{v}$ be a homomorphism of $V$ into End $\left(J,^{*}\right)$ such that (1)(a) $j B_{i} \in H_{y z}$ for $j \in J_{z}$ and $i \in I_{y}(b) J_{r} \beta_{b} \subseteq H_{b-1 r b}$ (2)(a) $g B e_{y}=g \beta_{y}=g^{*} e_{y}$ for $g \in J$ (b) $j B_{i}=j$ for $j \in H_{z}$ and $i \in I_{z}$. Let $(Y, I, J, V, B, \beta)$ denote $\{(i, a, j)$ : $a \in V, i \in I_{a a^{-1}}$, and $\left.j \in J_{a^{-1} a}\right\}$ under the multiplication (3) $(i, a, j)(u, b, z)=$ $\left(i \circ e_{a b(a b)-1}, a b, j B_{u} \beta_{b}^{*} z\right)$.

THEOREM 4.1. ( $Y, I, J, V, B, \beta)$ is a split extension of $T$ by $V$. Conversely, every such semigroup is isomorphic to some ( $Y, I, J, V$, $B, \beta)$. 
Proof. Let $S=(Y, I, J, V, B, \beta)$. If we let $g(c, d)=e_{(c d)-1}{ }_{c d}$ for all $c, d \in V$, it is easily verified that (1)-(4) of the definition of $(Y, I, J, V, B, \beta, g)$ are valid. Hence, $S$ is a standard regular semigroup by Theorem 3.14. Let $y_{0}$ denote the greatest element of $Y$. Using 2(a) and Lemmas 3.9 and 3.2, $\left(e_{y_{0}}, y_{0}, e_{y_{0}}\right) \in E\left(T_{y_{0}}\right)$. Using (4), $2(a)$ and the fact $(J, *)$ is locally inverse, $\left(e_{y_{0}}, y_{0}, e_{y_{0}}\right) S\left(e_{y_{0}}, y_{0}, e_{y_{0}}\right)=$ $\left\{\left(e_{a a^{-1}}, a, j\right): a \in V, j \in H_{a^{-1} a}\right\}$. Thus, using Lemmas 3.6,

$$
h_{a}=\left\{\left(e_{a a^{-1}}, a, j\right): j \in H_{a^{-1} a}\right\} \text {. }
$$

Let $v_{a}=\left(e_{a a^{-1}}, a, e_{a^{-1} a}\right)$. Hence, $v_{a} v_{b}=v_{a b}$. Thus, $S$ is a split extension of $T$ by $B$. Conversely, let $S$ be a standard regular semigroup which is a split extension of $T$ by $V$. Hence, using Lemma 2.17, $g(c, d)=e_{(c d)^{-1} c d}$ for all $c, d \in V$. Thus, using Theorem 3.14, $S=(Y, I, J, V, B, \beta, g)$ with $g(c, d)=e_{(c d)-1_{c d}}$. For brevity, let $(Y, I$, $J, V, B, \beta)=U$. Using 3(a), 1(a) and 1(b) of the definition of $S, c \rightarrow \beta_{c}$ defines a homomorphism of $V$ into End $\left(J,{ }^{*}\right)$, and (4) of the definition of $S$ reduces to (3) of the definition of $U$. Hence, $S \cong U$.

Let $A$ be an $\omega Y$-semilattice. Let $V=\left\{(n, k)_{\delta}: n, k \in N, \delta \in Y\right\}$ under the multiplication

$$
(n, k) \delta(r, s)_{\eta}=(n+r-\min (k, r), k+s-\min (k, r))_{f(k, r)}
$$

where $f(k, r)=\delta, \eta$, or $\delta \eta$ according to whether $k>r, r>k$, or $r=k$. Let $Y(=A), I, J, H$, and $\left\{e_{(k, \delta)}\right\}$ be as in the definition of $(Y, I, J, V, B, \beta)$. Let $(n, k)_{\dot{\delta}} \rightarrow \beta_{(n, k)_{\delta}}$ be a homomorphism of $V$ into End $\left(J,{ }^{*}\right)$ and let $i \rightarrow B_{i}$ be a homomorphism of $(I, \circ)$ into $P(J)$ such that 1(a) $j B_{i} \in H_{(n, \delta)(m, \lambda)}$ for $j \in J_{(m, \lambda)}$ and $i \in I_{(n, \delta)} 1(\mathrm{~b}) \quad J_{(r, \eta)}, \beta_{(n, k)_{o}} \leqq$ $H_{(r+k-\min (r, n), f(r, n))}$. 2(a) $h B_{e_{(n, \delta)}}=h \beta_{(n, \delta)}=h^{*} e_{(n, \delta)}$ for $h \in J$ (b) $j B_{i}=j$ if $j \in H_{(n, \delta)}$ and $i \in I_{(n, \delta)}$. Let $I_{\delta}=\bigcup\left(I_{(n, \delta)}: n \in N\right)$ and let $J_{\delta}=$ $\mathrm{U}\left(J_{(n, \delta)}: n \in N\right)$. Let $(\omega Y, I, J, B, \beta)$ denote $\bigcup\left(I_{\delta} \times J_{\delta}: \delta \in Y\right)$ under the multiplication (3): if $i \in I_{(n, \delta)}, j \in J_{(k, \delta)}, u \in I_{(r, \eta)}$, and $z \in J_{(s, \eta)}$, $(i, j)(u, z)=\left(i \circ e_{(n+r-\min (k, r), f(k, r))}, j B_{u} \beta_{(r, s)_{\eta}}^{*} z\right)$.

THEOREM 4.2. ( $(\omega Y, I, J, B, \beta)$ is a standard regular semigroup of type $\omega Y$, and conversely every such semigroup is isomorphic to some $(\omega Y, I, J, B, \beta)$.

Proof. Let $S$ be a standard regular semigroup of type $\omega Y$. Using Lemmas 2.1 and 2.2, $E\left(S_{0}\right)=\left\{e_{(n, \tilde{o})}: n \in N, \delta \in Y\right\} \cong A$. Furthermore, $e_{(n, \delta)} \mathscr{D} e_{(m, \lambda)}\left(\varepsilon S_{0}\right)$ if and only if $\delta=\lambda$. Hence, use [15, Lemma 2.1, Theorem 2.3, and Corollaries 2.2 and 2.4] to show $S$ is a split extension of $T$ by $V$ (given in the definition of $(\omega Y, I, J, B, \beta)$ ). Hence, $S \cong(A, I, J, V, B, \beta)$ by Theorem 4.1. Using [15, Corollary 
2.4], $J_{(r, \eta)} \beta_{(n, k)_{\delta}} \subseteq H_{(r+k-\min (r, n), f(r, n))}$ and $\left((n, k)_{\delta}(r, s)_{\eta}\right)\left((n, k)_{\delta}(r, s)_{\eta}\right)^{-1}=$ $(n+r-\min (k, r), f(k, r))$. Hence, using Theorem 4.1 and [15, Corollary 2.4], $S \cong\left\{\left(i,(n, k)_{\delta}, j\right):(n, k)_{\delta} \in V, i \in I_{(n, \delta)}, J \in J_{(k, \delta)}\right\}$ under the multiplication $\left(i,(n, k)_{j}, j\right)\left(u,(r, s)_{\eta}, z\right)=\left(i_{\circ} e_{(n+r-\min (k, r), f(k, r))},(n, k)_{\delta}(r, s)_{\eta}\right.$, $\left.j B_{u} \beta_{(r, s)_{\eta}}{ }^{*} z\right)$. Hence, $\left(i,(n, k)_{\delta}, j\right) \zeta=(i, j)$ defines an isomorphism of $(A, I, J, V, B, \beta)$ onto $(\omega, Y, I, J, B, \beta)$. Conversely, consider $S=$ $(\omega Y, I, J, B, \beta)$. Using [15, Theorem 2.3 and Corollary 2.4], $V$ is an inverse semigroup with semilattice of idempotents $A$ and each $\mathscr{H}$ class of $V$ consists of a single element. Using [15, Corollary 2.4], 1 (b) of the definition of $(A, I, J, V, B, \beta)$ is valid. Hence, $\zeta^{-1}$ defines an isomorphism of $S$ onto $(A, I, J, V, B, \beta)$. By the proof of Theorem 4.1, $(A, I, J, V B, \beta)=(A, I, J, V, B, \beta, g)$ with $\left.g(c, d)=e_{(c d)-1}\right)_{c d}$. Hence, using [15, Corollary 2.4] and Lemmas 3.2, 3.7, and 3.9-3.12, $S$ is a standard regular semigroup of type $\omega Y$.

THEOREM 4.3. $S$ is a simple standard regular semigroup of type $\omega$ if and only if $S$ is a standard regular semigroup of type $\omega Y$ with $Y$ a finite chain $0>1>2>\cdots d-1$ where $d$ is a positive integer.

Proof. Let $S$ be a simple standard regular semigroup of type $\omega$. Hence, $S_{0}$ is a simple semigroup. Thus, using Lemma 2.15 and [15, Lemma 7.5 and Theorem 2.3], $V$ is the semigroup described in the definition of $(\omega Y, I, J, B, \beta)$ with $Y$ the finite chain $0>1>2$ $\cdots>d-1$ where $d$ is a positive integer. Hence, using Theorem 3.14 (and its proof), [15, Corollary 2.4], Lemmas 3.2, 3.7, and 3.9-3.12, $S$ is a standard regular semigroup of type $\omega Y$ with $Y$ the finite chain $0>1>2 \cdots>d-1$ where $d$ is a positive integer. Conversely, let $S$ be a standard regular semigroup of type $\omega Y$ with $Y$ a finite chain. It is easily seen that $S$ is a standard regular semigroup of type $\omega$. We next show that any standard regular semigroup of type $\omega Y$ is simple. Let $S=(\omega Y, I, J, B, \beta)$. Let $i \in I_{(n, \delta)}, j \in J_{(k, \delta)}, u \in I_{(r, \eta)}$, and $v \in J_{(s, \eta)}$. Let $q=e_{(n+1, \eta)} B_{i} \beta_{(n+1, s) \eta}^{*} j \beta_{(k+1, s)}$. Using (1), $q \in H_{(s, \eta)}$. Hence, since $J_{(s, \eta)}$ is a right group, there exists $b \in J_{(s, \eta)}$ such that $q^{*} b=v$. Thus, (3), (2(a)), the fact $c \rightarrow \beta_{c}$ is a homomorphism of $V$ into End $(J, *)$, and $\left.(1),\left(u, e_{(n+1), \eta}\right)(i, j)\left(e_{(k+1, \eta)}, b\right)\right)=(u, v)$.

If $A$ is a finite set, $|A|$ will denote the number of elements of $A$.

THEOREM 4.4. $S$ is a standard regular bisimple semigroup of type $\omega$ if and only if $S$ is a standard regular semigroup of type $\omega Y$ with $|Y|=1$.

Proof. Apply Theorem 4.3, Lemma 3.7, and [15, Corollary 2.4]. 
5. Some other classes of standard regular semigroups, A regular semigroup $S$ is termed standard orthodox ( $\mathscr{L}$-unipotent) if $T$ is a locally inverse semilattice $Y$ of rectangular groups (right groups) $\left(T_{y}: y \in Y\right)\left(\left(J_{y}: y \in Y\right)\right)$ where $Y$ has a greatest element. An inverse semigroup $S$ is termed standard inverse if $T$ is a semilattice $Y$ of groups $\left(H_{y}: y \in Y\right)$ where $Y$ has a greatest element. In this section, we give structure theorems for these classes of semigroups.

Let $Y, V, I, J,\left\{e_{k}\right\}, H, \beta$, and $g$ be as in the definition of $(Y, I, J$, $V, B, \beta, g)$. Furthermore, assume that $1(\mathrm{~b}), 1(\mathrm{c}), 2(\mathrm{c}), 3(\mathrm{a})$, and $3(\mathrm{~b})$ of that definition are valid and that 2(a) $g \beta_{y}=g^{*} e_{y}$ for $y \in Y$ and $g \in J$. Let $(Y, I, J, V, \beta, g)$ denote $\left\{(i, a, j): a \in V, i \in I_{a a^{-1}}, j \in J_{a^{-1} a}\right\}$ under the multiplication (4)

$$
(i, a, j)(u, b, z)=\left(i \circ e_{(a b)(a b)-1}, a b, g(a, b)^{*} j \beta_{b}^{*} z\right) .
$$

REMARK 5.1. ( $Y, I, J, V, \beta, g)$ is a standard orthodox semigroup and conversely every standard orthodox semigroup is isomorphic to some $(Y, I, J, V, \beta, g)$.

Proof. Let $S$ be a standard orthodox semigroup. It is easily seen that $S$ is standard regular. We apply Theorem 3.14. Let $j \in J$ and $u \in I$. By the proof of Lemma 2.11, $j B_{u}=j u$. Let $\left\{\zeta_{y, z}: y z \in Y\right\}$ denote the set of structure homomorphisms of $T$. Let $j \in J_{y}$ and $u \in I_{z}$. Hence, using the fact that $T_{y z}$ is a rectangular group, $j u=j \zeta_{y, y z} u \zeta_{z, y z}=$ $j \zeta_{y, y z} e_{z} \zeta_{z, y z}=j e_{z}$. Hence $j B_{u}=j^{*} e_{z}$. Let $j \in J, u \in I_{b b^{-1}}$, and $z \in J_{b^{-1} b}$. Hence, using the fact $\beta_{b} \in$ End $\left(J,{ }^{*}\right)$ and $1(b), j B_{u} \beta_{b}^{*} z=\left(j^{*} e_{b b}\right) \beta_{b}^{*} z=$ $j \beta_{b}^{*} e_{b-1}^{*} z=j \beta_{b}^{*} z$. Thus, (4) of Theorem 3.14 reduces to (4) in the definition of $(Y, I, J, V, \beta, g)$.

Conversely, we show $(Y, I, J, V, \beta, g)$ is a standard orthodox semigroup. First, we apply Theorem 3.14 to show that $(Y, I, J, V, \beta, g)$ is a standard regular semigroup. We define $j B_{i}=j^{*} e_{y}$ for $j \in J$ and $i \in I_{y} \quad$ Let $u \in I_{y}, v \in I_{z}$, and $j \in J$. Hence $j B_{u} B_{v}=j e_{y}^{*} e_{z}=j^{*} e_{y_{z}}=$ $j B_{u \circ v}$. Let $j, h \in J$ and $i \in I_{y}$. Hence, $\left(j^{*} h\right) B_{i}=\left(j^{*} h\right)^{*} e_{y}=j^{*}\left(h^{*} e_{y}\right)=$ $j^{*}\left(h B_{i}\right)$. Thus, $i \rightarrow B_{i}$ is a homomorphism of $(I, o)$ into $P(J, *)$. Let $j \in J_{y}$ and $i \in I_{z}$. Let $\left\{\gamma_{y, z}: y, z \in Y\right\}$ denote the set of structure homomorphisms of $(J, *)$. Hence, for $i \in I_{z}, j B_{i}=j^{*} e_{z}=j \gamma_{y, y_{z}}^{*} e_{z} \gamma_{z, y z}$. However, $e_{z} \gamma_{z, y z} \leqslant e_{z}$, and $e_{y z} \leqslant e_{z}$. Hence, using the fact that $\left(J,{ }^{*}\right)$ is locally inverse and $J_{y z}$ is a right group $e_{z} \gamma_{z, z y}=e_{y z}$. Thus, $j B_{i}=j \gamma_{y, y z}^{*} e_{y z} \in$ $H_{y_{z}}$. Hence, 1(a) of Theorem 3.14 is valid. By definition, $j B_{e_{y}}=$ $j^{*} e_{y}$. If $j \in H_{y}$ and $i \in I_{y}, j B_{i}=j^{*} e_{y}=j$. Hence, 2(b) of Theorem 3.14 is valid. Let $j \in J, u \in I_{b b^{-1}}$, and $z \in J_{b^{-1} b}$. As above, $j \beta_{b}^{*} z=$ $j B_{u} \beta_{b}^{*} z$. Thus, (4) of Theorem 3.14 and (4) of the definition of ( $Y$, $I, J, V, \beta, g)$ are equivalent. Hence, using Theorem 3.14, $(Y, I, J$, $V, \beta, g)$ is a standard regular semigroup. Let $(i, y, j),(u, y, z) \in T_{y}$ 
(note, Lemma 3.9). Hence, using 2(c) and 2(a), $(i, y, j)(u, y, z)=$ $\left(i, y, j^{*} z\right)$. Thus, using [1, Theorem 1.27], $T_{y}$ is a rectangular group. Hence, using Lemmas 3.9-3.11, $(Y, I, J, V, \beta, g)$ is a standard orthodox semigroup.

REMARK 5.2. By a result of Preston, Yamada, and Clifford, [2, Proposition 1], if $T$ is a semilattice of rectangular groups, $T$ is an orthodox semigroup. Conversely, every Cliffordian semigroup which is orthodox is a semilattice of rectangular groups.

Let $Y, V, J$, and $\beta$ be as in Theorem 5.1. For each $y \in Y$, select $e_{y} \in E\left(J_{y}\right)$ such that $e_{y}^{*} e_{z}=e_{y z}$ for all $y, z \in Y$. Let $H_{y}$ denote the maximal subgroup of $J_{y}$ containing $e_{y}$. Let $g$ be as in Theorem 5.1 and assume 1(b), 1(c), 2(a), 2(c), 3(a), and $3(\mathrm{~b})$ of Theorem 5.1 are valid. Let $(Y, J, V, \beta, g)$ denote $\left\{(\alpha, j): a \in V, j \in J_{a^{-1} a}\right\}$ under the multiplication $(4)(a, j)(b, z)=\left(a b, g(a, b)^{*} j \beta_{b}^{*} z\right)$.

THEOREM 5.3. ( $Y, J, V, \beta, g)$ is a standard $\mathscr{L}$-unipotent semigroup and, conversely, every standard $\mathscr{L}$-unipotent semigroup is isomorphic to some $(Y, J, V, \beta, g)$.

Proof. Let $S$ be a standard $\mathscr{L}$-unipotent semigroup. Hence, using [1, Theorem 1.27], $S$ is standard orthodox. Using Theorem 5.1, $S=(Y, I, J, V, \beta, g)$. Let $i \in I_{y}$. Using Lemma 3.2 and the proof of Lemma 5.1, $\left(i, y, e_{y}\right),\left(e_{y}, y, e_{y}\right) \in E(S)$. Hence, using Lemma 3.5 and the fact each $\mathscr{L}$-class of $S$ contains precisely one idempotent, $i=e_{y}$. Thus, $I_{y}=\left\{e_{y}\right\}$ for each $y \in Y$. Hence, $\left(e_{a a^{-1}}, a, j\right) \phi=(a, j)$ defines an isomorphism of $(Y, I, J, V, \beta, g)$ onto $(Y, J, V, \beta, g)$. Conversely, we show that $S=(Y, J, V, \beta, g)$ is a standard $\mathscr{L}$-unipotent semigroup. Let $I_{y}=\left\{e_{y}\right\}$. Define $e_{y} \circ e_{z}=e_{y z}$ and let $I=\bigcup\left(I_{y}: y \in Y\right)$. Hence, $(I, \circ)$ is a standard regular semilattice $Y$ of left zero semigroups $\left(I_{y}: y \in Y\right)$. Then, $\phi^{-1}$ is an isomorphism of $S$ onto $(Y, I, J$, $V, \beta, g)$. Hence, $S$ is a standard orthodox semigroup. Using Lemmas 3.2 and 3.5 , each $\mathscr{L}$-class of $S$ contains precisely one idempotent. Hence, it easily follows that $S$ is standard $\mathscr{L}$-unipotent.

REMARK 5.4. A semigroup $S$ is termed $\mathscr{L}$-unipotent if each $\mathscr{L}$-class of $S$ contains precisely one idempotent [13]. Hence, a standard regular semigroup is $\mathscr{L}$-unipotent in the sense of [13] if and only if it is standard $\mathscr{L}$-unipotent.

Let $Y, V$, and $\beta$ be as in Theorem 5.3. Let $(H, *)$ be a semilattice $Y$ of groups $\left(H_{y}: y \in Y\right)$ and let $e_{y}$ denote the identity of $H_{y}$ and let $g$ be as in Theorem 5.3 and assume 1(b), 1(c), 2(a), 2(c), 3(a) and $3(\mathrm{~b})$ of Theorem 5.3 are valid. Let $(Y, H, V, \beta, g)$ denote $\{(a, j)$ : $\left.a \in V, j \in H_{a^{-1} a}\right\}$ under the multiplication (4) 


$$
(a, j)(b, z)=\left(a b, g(a, b)^{*} j \beta_{b}^{*} z\right)
$$

THEOREM 5.5. ( $Y, H, V, \beta, g)$ is a standard inverse semigroup, and conversely every standard inverse semigroup is isomorphic to some $(Y, H, V, \beta, g)$.

Proof. Let $S$ be a standard inverse semigroup. Then, $S$ is standard $\mathscr{L}$-unipotent. Hence, using Theorem 5.3, $S \cong(Y, J, V$, $\beta, g)$. Using Lemma 3.2, $E(Y, J, V, \beta, g)=\left\{(y, j) ; y \in Y, j \in E\left(J_{y}\right)\right\}$. Let $j \in E\left(J_{y}\right)$. Hence, using 2(c) and 2(a), $(y, j)\left(y, e_{y}\right)=\left(y, e_{y}\right)$ and $\left(y, e_{y}\right)(y, j)=(y, j)$. Thus, $j=e_{y}$. Hence, $J_{y}=H_{y}$ for all $y \in Y$. Thus, $S \cong(Y, H, V, \beta, g)$. Conversely, let $S=(Y, H, V, \beta, g)$. Since $e_{y}^{*} e_{z}=e_{y, z}, S$ is standard $\mathscr{L}$-unipotent by Theorem 5.3. Using Lemma 3.2, $E(S)=\left\{\left(y, e_{y}\right): y \in Y\right.$. $\}$ Hence, $E(S)$ is a semilattice. Thus, it is easily seen that $S$ is a standard inverse semigroup.

REMARK 5.6. A characterization of standard orthodox semigroups may be obtained by combining a theorem of M. Yamada [18, Theorem 2] with Theorem 5.5.

6. The congruence $t$. Let $S$ be a standard regular semigroup. Let $t=\left\{(a, b) \in S^{2}: a a^{\prime}, b b^{\prime} \in E\left(T_{y}\right)\right.$ and $a^{\prime} a, b^{\prime} b \in E\left(T_{z}\right)$ for some $a^{\prime} \in$ $\mathscr{F}(a), b^{\prime} \in \mathscr{F}(b)$, and $\left.y, z \in Y\right\}$. We introduced $t$ in a special case in [12] and used it in subsequent papers (see [15] and [16] for example). We show $t$ is a congruence on $S, S / t \cong V$, and ker $t$ (the collection of $t$-classes of $S$ containing idempotents) $=T$. We note that $S$ is a regular extension of $T$ by $V$ in the sense of Yamada [19].

\section{LEMMA 6.1. $\mathscr{I}((i, a, j)) \cap\left(I_{a^{-1} a} \times\left\{a^{-1}\right\} \times J_{a a^{-1}}\right) \neq \square$.}

Proof. Let $y=a a^{-1}$ and let $u \in I_{a^{-1} a}$. Hence, using (1), $g\left(a, a^{-1}\right)^{*} j B_{u} \beta_{a^{-1}} \in H_{a a^{-1}}$ for $j \in J_{a^{-1} a}$. There exists $v \in H_{a a^{-1}}$ such that $g\left(a, a^{-1}\right)^{*} j B_{u} \beta_{a^{-1}}^{*} v=e_{y}$. Hence, using (4), $(i, a, j)\left(u, a^{-1}, v\right)=\left(i, y, e_{y}\right)$. However, using $2(\mathrm{~b})$, Lemmas $3.2,3.3$, and 3.5,

$$
(i, a, j)=\left(i, y, e_{y}\right)(i, a, j)=(i, a, j)\left(u, a^{-1}, v\right)(i, a, j)
$$

while $\left(u, a^{-1}, v\right)(i, a, j)\left(u, a^{-1}, v\right)=\left(u, a^{-1}, v\right)\left(i, y, e_{y}\right)=\left(u, a^{-1}, v\right)$.

Lemma 6.2. $(i, a, j) t(u, b, v)$ if and only if $a=b$.

Proof. Using Lemmas 6.1 and 3.9, there exists $(i, a, j)^{\prime} \in \mathscr{F}(i, a, j)$ and $(u, b, v)^{\prime} \in \mathscr{J}(u, b, v)$ such that $(i, a, j)(i, a, j)^{\prime} \in T_{a a^{-1}},(u, b, v)(u, b, v)^{\prime} \in$ $T_{b^{-1}},(i, a, j)^{\prime}(i, a, j) \in T_{a^{-1} a}$, and $(u, b, v)^{\prime}(u, b, v) \in T_{b^{-1} b_{b}}$. Hence, 
$(i, a, j) t(u, b, v)$ if and only if $a a^{-1}=b b^{-1}$ and $a^{-1} a=b^{-1} b$.

THEOREM 6.3. $t$ is a congruence relation on $S, S / t \cong V$, and ker $t=T$.

Proof. The first two assertions are easily seen. Using Lemmas $3.2,3.9$, and 3.10 , $\operatorname{ker} t=T$.

REMARK 6.4. Using Lemmas 3.9-3.11 and the fact $(i, a, j) \phi=a$ defines a homomor phism of $S$ onto $V, S$ is a regular extension of $T$ by $V$ in the sense of Yamada [19, page 4]. Thus, using Theorem 6.3, $S$ is a regular extension of $T$ by $S / t$.

\section{REFERENCES}

1. A. H. Clifford and G. B. Preston, The algebraic theory of semigroups, Math. Surveys of the Amer. Math. Soc. 7, Vol. 1, Providence, R. I., 1961; Vol. 2, Providence, R. I., 1967.

2. A. H. Clifford, The structure of orthodox unions of groups, Semigroup Forum, 3 (1972), 283-337.

3. T. E. Hall, On regular semigroups whose idempotents form a semigroup, Bull. Australian Math. Soc., 1 (1969), 195-208.

4. J. W. Hogan, Bisimple semigroups with idempotents well ordered, Semigroup Forum, 6 (1973), 298-316.

5. W. D. Munn, On simple inverse semigroups, Semigroup Forum, 1 (1970), 63-74.

6. —, Fundamental inverse semigroups, Quarterly J. Math. Oxford, 21 (1970), $157-170$.

7. R. J. Warne, Extensions of completely 0-simple semigroups by completely 0-simple semigroups, Proc. Amer. Math. Soc., 17 (1966), 524-526.

8. — A class of bisimple inverse semigroups, Pacific J. Math., 18 (1966), 563577.

9. - The idempotent separating congruences of a bisimple inverse semigroup with identity, Publicationes Mathematicae, 13 (1966), 203-206.

10. - Simple L-regular semigroups, The Formosan Science, 24 (1970), 71-77.

11. - A comment on "Simple L-regular semigroups," The Formosan Science, 24 (1970), 78.

12. — E-bisimple semigroups, J. Natural Sci. and Math. (Lahore), 11 (1971), $51-81$.

13. - $L$ L-unipotent semigroups, Nigerian J. Science, 5 (1972), 245-248.

14. - On the structure of semigroups which are unions of groups, Trans. Amer. Math. Soc., 186 (1973), 385-401.

15. ——, — $\omega Y$-L L-unipotent semigroups, Jnānabha, 3 (1973), 99-118.

16. — Generalized $\omega$ - L $\mathscr{L}$-unipotent bisimple semigroups, Pacific J. Math., 51 (1974), 631-648.

17. M. Yamada and N. Kimura, Note on idempotent semigroups, II, Proc, Japan Acad., 34 (1958), 110-112,

18. Miyuki Yamada, Regular semigroups whose idempotents satisfy permutation identities, Pacific J. Math., 21 (1967), 371-392.

19. - On regular extensions of a semigroup which is a semilattice of completely simple semigroups, Mem. Fac. Lit. and Sci. Shimane Univ. Nat. Sci., 7 (1974), $1-17$. 
Received May 23, 1975 and in revised form April 12,1976. An abstract of the major result of section 1 appeared in Notices Amer. Math. Soc. 20 (1973), A-561 under the title Locally inverse unions of groups. Abstracts of the remainder of the paper appeared in the Notices of the Amer. Math. Soc. 22 (1975), No. 7, A-704, 75T-A258 and 23 (1976), No. 2, A-265, 76T-A34 under the titles Strictly regular semigroups and Strictly regular semigroups, II, respectively.

UNiversity of Alabama in Birmingham 


\section{PACIFIC JOURNAL OF MATHEMATICS}

\section{EDITORS}

RICHARD ARENS (Managing Editor)

University of California

Los Angeles, California 90024

\section{R. A. Beaumont}

University of Washington

Seattle, Washington 98105
J. DugundjI

Department of Mathematics

University of Southern California

Los Angeles, California 90007

D. Gilbarg and J. Milgram

Stanford University

Stanford, California 94305

\section{ASSOCIATE EDITORS}
E. F. BECKENBACH
B. H. NeumanN
F. WOLF
K. YosHIDA

\section{SUPPORTING INSTITUTIONS}

\author{
UNIVERSITY OF BRITISH COLUMBIA \\ CALIFORNIA INSTITUTE OF TECHNOLOGY \\ UNIVERSITY OF CALIFORNIA \\ MONTANA STATE UNIVERSITY \\ UNIVERSITY OF NEVADA \\ NEW MEXICO STATE UNIVERSITY \\ OREGON STATE UNIVERSITY \\ UNIVERSITY OF OREGON \\ OSAKA UNIVERSITY
}

\author{
UNIVERSITY OF SOUTHERN CALIFORNIA \\ STANFORD UNIVERSITY \\ UNIVERSITY OF HAWAII \\ UNIVERSITY OF TOKYO \\ UNIVERSITY OF UTAH \\ WASHINGTON STATE UNIVERSITY \\ UNIVERSITY OF WASHINGTON \\ AMERICAN MATHEMATICAL SOCIETY
}

The Supporting Institutions listed above contribute to the cost of publication of this Journal, but they are not owners or publishers and have no responsibility for its content or policies.

Mathematical papers intended for publication in the Pacific Journal of Mathematics should be in typed form or offset-reproduced, (not dittoed), double spaced with large margins. Please do not use built up fractions in the text of your manuscript. You may however, use them in the displayed equations. Underline Greek letters in red, German in green, and script in blue. The first paragraph or two must be capable of being used separately as a synopsis of the entire paper. Items of the bibliography should not be cited there unless absolutely necessary, in which case they must be identified by author and Journal, rather than by item number. Manuscripts, in triplicate, may be sent to any one of the editors. Please classify according to the scheme of Math. Reviews, Index to Vol. 39. All other communications should be addressed to the managing editor, or Elaine Barth, University of California, Los Angeles, California, 90024.

The Pacific Journal of Mathematics expects the author's institution to pay page charges, and reserves the right to delay publication for nonpayment of charges in case of financial emergency.

100 reprints are provided free for each article, only if page charges have been substantially paid. Additional copies may be obtained at cost in multiples of 50 .

The Pacific Journal of Mathematics is issued monthly as of January 1966. Regular subscription rate: $\$ 72.00$ a year (6 Vols., 12 issues). Special rate: $\$ 36.00$ a year to individual members of supporting institutions.

Subscriptions, orders for back numbers, and changes of address should be sent to Pacific Journal of Mathematics, 103 Highland Boulevard, Berkeley, California, 94708.

PUBLISHED BY PACIFIC JOURNAL OF MATHEMATICS, A NON-PROFIT CORPORATION

Printed at Kokusai Bunken Insatsusha (International Academic Printing Co., Ltd.), 8-8, 3-chome, Takadanobaba, Shinjuku-ku, Tokyo 160, Japan. 


\section{Pacific Journal of Mathematics \\ Vol. 65, No. $2 \quad$ October, 1976}

Andrew Adler, Weak homomorphisms and invariants: an example .......... 293

Howard Anton and William J. Pervin, Separation axioms and metric-like

functions ............................................. 299

Ron C. Blei, Sidon partitions and p-Sidon sets .................... 307

T. J. Cheatham and J. R. Smith, Regular and semisimple modules ........... 315

Charles Edward Cleaver, Packing spheres in Orlicz spaces .............. 325

Le Baron O. Ferguson and Michael D. Rusk, Korovkin sets for an operator on a

space of continuous functions ............................. 337

Rudolf Fritsch, An approximation theorem for maps into Kan fibrations ....... 347

David Sexton Gilliam, Geometry and the Radon-Nikodym theorem in strict

Mackey convergence spaces .................................

William Hery, Maximal ideals in algebras of topological algebra valued

functions ...................................... 365

Alan Hopenwasser, The radical of a reflexive operator algebra ........... 375

Bruno Kramm, A characterization of Riemann algebras................. 393

Peter K. F. Kuhfittig, Fixed points of locally contractive and nonexpansive

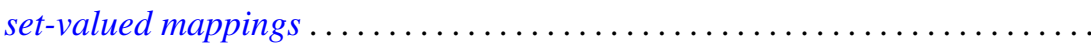

Stephen Allan McGrath, On almost everywhere convergence of Abel means of

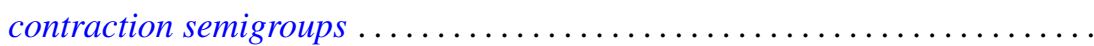

Edward Peter Merkes and Marion Wetzel, A geometric characterization of

indeterminate moment sequences............................ 409

John C. Morgan, II, The absolute Baire property ................... 421

Eli Aaron Passow and John A. Roulier, Negative theorems on generalized convex approximation .................................... 437

Louis Jackson Ratliff, Jr., A theorem on prime divisors of zero and characterizations of unmixed local domains ..............

Ellen Elizabeth Reed, A class of $T_{1}$-compactifications................... 471

Maxwell Alexander Rosenlicht, On Liouville's theory of elementary

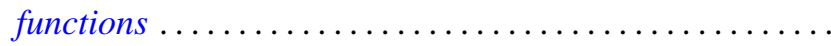

Arthur Argyle Sagle, Power-associative algebras and Riemannian

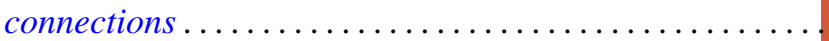

Chester Cornelius Seabury, On extending regular holomorphic maps from Stein manifolds...

Elias Sai Wan Shiu, Commutators and numerical ranges of powers of operators ...................................

Donald Mark Topkis, The structure of sublattices of the product of $n$ lattices ... 525

John Bason Wagoner, Delooping the continuous $K$-theory of a valuation

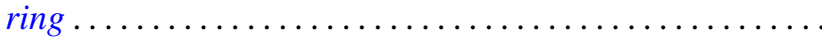

Ronson Joseph Warne, Standard regular semigroups...........

Anthony William Wickstead, The centraliser of $E \otimes_{\lambda} F \ldots$. 